\title{
A FREE PRODUCT FORMULA FOR THE SOFIC DIMENSION
}

\author{
ROBERT GRAHAM AND MIKAEL PICHOT
}

\begin{abstract}
It is proved that if $G=G_{1} *_{G_{3}} G_{2}$ is free product of probability measure preserving $s$-regular ergodic discrete groupoids amalgamated over an amenable subgroupoid $G_{3}$, then the sofic dimension $s(G)$ satisfies the equality$$
s(G)=\mathfrak{h}\left(G_{1}^{0}\right) s\left(G_{1}\right)+\mathfrak{h}\left(G_{2}^{0}\right) s\left(G_{2}\right)-\mathfrak{h}\left(G_{3}^{0}\right) s\left(G_{3}\right)
$$

where $\mathfrak{h}$ is the normalized Haar measure on $G$.

Let $G$ be a group. The sofic dimension of $G$ is an asymptotic invariant that accounts for the number of unital maps

$$
\sigma: F_{ \pm}^{n} \rightarrow \operatorname{Sym}(d)
$$

from the "Cayley ball" $F_{ \pm}^{n}$ of radius $n$ in $G$ into the symmetric group $\operatorname{Sym}(d)$, where $F \subset G$ is a finite set, $n$ is an integer, $d$ is a 'very large' integer and the maps $\sigma$ are multiplicative and free up to an error $\delta>0$ relative to the normalized Hamming distance on $\operatorname{Sym}(d)$ (see $\$ 1$ below). If $\operatorname{SA}(F, n, \delta, d)$ is the (finite) set of all such maps, and NSA $:=\left|\left\{\sigma_{\mid F}, \sigma \in \mathrm{SA}\right\}\right|$, the sofic dimension of $F$ is:

$$
s(F)=\inf _{n \in \mathbb{N}} \inf _{\delta>0} \limsup _{d \rightarrow \infty} \frac{\log \operatorname{NSA}(F, n, \delta, d)}{d \log d}
$$

(so the limit is on $d$ first, and then on $\delta$ and $n$ ). This definition was considered in [DKP1] and DKP2]. It is a combinatorial version of Voiculescu's (microstate) free entropy dimension $\delta(F)$, which can be defined by a similar formula involving maps

$$
\sigma: F_{ \pm}^{n} \rightarrow U(d)
$$

into the unitary group $U(d)$ (see [Voi96, Jun1]). It can be shown that the value of $s(F)$ doesn't dependent on the finite generating set $F$ of $G$ and is therefore denoted $s(G)$. A limiting process allows to define of $s(G)$ for an arbitrary group $G$.

The definition of the sofic dimension can be extended to probability measure preserving (pmp) actions of countable groups, their orbit equivalence relations, and more generally to discrete pmp groupoids. We refer to [DKP2, Definition 2.3] for the general groupoid definition. An interesting feature of $s$ is to provide combinatorial proofs of statements in orbit equivalence theory (for example, Corollary 7.5 in [DKP1] reproves Gaboriau's theorem that the free groups on $p$ generators are pairwise non orbit equivalent using the counting method).

Let $G$ be a pmp groupoid and assume that $G=G_{1} *_{G_{3}} G_{2}$ is an amalgamated free product over a subgroupoid $G_{3}$. A free product formula of the form

$$
s(G)=s\left(G_{1}\right)+s\left(G_{2}\right)-s\left(G_{3}\right)
$$

is known to hold in the following cases (under some technical assumptions, for example "finitely generated" and/or " $s$-regularity"):

(1) $G, G_{1}, G_{2}$, and $G_{3}$ are a pmp equivalence relations on $(X, \mu)$ and $G_{3}$ is amenable as an equivalence relation: see [DKP1, Theorem 1.2]. 
(2) $G, G_{1}, G_{2}$, and $G_{3}$ are countable groups and $G_{3}$ is an amenable group: see DKP2, Theorem 4.10].

(3) $G$ is the crossed product groupoid $G:=G_{1} \star G_{2} \ltimes X$ of a pmp action $\left(G_{1} *\right.$ $\left.G_{2}\right) \curvearrowright(X, \mu)$, where $G_{1}$ and $G_{2}$ are countable groups and $X$ is a standard probability space. Here $G_{3}$ is assumed to be the trivial group but the action of $G_{1} * G_{2}$ is not necessarily free (if free this is covered by (1)): see [DKP2, Theorem 6.4].

The general strategy to establish this sort of formula was devised by Voiculescu for the free entropy dimension: see in particular [Voi91, Voi96, Voi98].

The proofs of the above results in DKP1, DKP2] apply distinct tools to handle the amenable amalgamated part, namely the Connes-Feldmann-Weiss theorem in (1), and the Ornstein-Weiss quasi-tiling theorem in (2). This was a reason why it was hardly conceivable to incorporate an amenable amalgamated subgroup $G_{3}$ in (3); in fact, the technical details would presumably (to quote [DKP2, §6]) be 'formidable' even if the action of $G_{3} \curvearrowright X$ is essentially free.

We follow a different approach here, based on the use of Bernoulli shifts as a "correspondence principle"

$$
\text { groupoids } \rightsquigarrow \text { equivalence relations }
$$

by which we mean that proving a result for (pmp) equivalence relations 'automatically' implies an a priori more general statement for ( $\mathrm{pmp}$ ) groupoids in a variety of situation, and in particular for the computation of $s$ (see $\$ 9$ for more details).

The exact assumptions that we need for the free product formula are described in the following statement, which is the main result of this paper:

Theorem 1. Let $G$ be a discrete pmp groupoid of the form $G=G_{1} *_{G_{3}} G_{2}$, where $G_{1}, G_{2}$ are s-regular ergodic subgroupoids of $G$ and $G_{3}$ is an amenable groupoid, then

$$
s(G)=\mathfrak{h}\left(G_{1}^{0}\right) s\left(G_{1}\right)+\mathfrak{h}\left(G_{2}^{0}\right) s\left(G_{2}\right)-\mathfrak{h}\left(G_{3}^{0}\right) s\left(G_{3}\right)
$$

where $\mathfrak{h}$ is the normalized Haar measure on $G$ and $G^{0}$ is the object space of $G$.

The more technical assumptions in this result can be weakened slightly. For example, one way to remove the $s$-regularity assumption, following Voiculescu's idea, see e.g. [Voi98, Remark 4.8], is to replace the limsup in the definition of $s(F)$ by a limit along a fixed ultrafilter $\omega$. What is rather unclear is the extend to which the assumption that $G_{3}$ is amenable is essential. Cohomological tools can be used to prove a similar formula for the first $L^{2}$ Betti number $\beta_{1}$ under the much weaker assumption that $\beta_{1}\left(G_{3}\right)=0$. (This is a result of Lück see BDJ, Theorem A.1] for the group case.) Furthermore Mineyev and Shlyakhtenko MS have shown that Voiculescu's 'non-microstate' free entropy dimension $\delta^{*}$ satisfies $\delta^{*}(G)=\beta_{1}(G)-\beta_{0}(G)+1$ for any finitely generated group $G$, and therefore we have the formula

$$
\delta^{*}\left(G_{1} *_{G_{3}} G_{2}\right)=\delta^{*}\left(G_{1}\right)+\delta^{*}\left(G_{2}\right)-\delta^{*}\left(G_{3}\right)
$$

where $G_{1}$ and $G_{2}$ are finitely generated groups and $G_{3}$ is a group such that $\beta_{1}\left(G_{3}\right)=0$. A fundamental relation between the microstate and the non-microstate approach to free entropy is provided by the Biane-Capitaine-Guionnet inequality $\delta \leq \delta^{*}$ [BCG]. A free product formula for $\delta_{0}$ has been established in [BDJ] for amalgamation of ( $\delta_{0}$-regular) groups over an amenable subgroup (where $\delta_{0} \leq \delta$ is a technical modification of $\delta$ not depending on the generating set of the group, 
see [Voi96, Section 6] and [Voi98]). We also note that the above correspondence principle for $\delta_{0}$ is probably less useful as the amenable part can always be handled uniformly using the hyperfiniteness of von Neumann algebra $L G_{3}$ (see in particular Jun2]; for example, the proof in BDJ] in the group case doesn't rely on quasi-tilings). Concerning pmp equivalence relations, a free product formula has been established by Gaboriau Gab] for the cost, allowing for amalgamations over amenable subrelations, and by Shlyakhtenko Shl] for $\delta_{0}$, for free product with trivial amalgamation.

Question 2. Can the assumption that $G_{3}$ is amenable in Theorem 1 be weakened (for example to $\beta_{1}\left(G_{3}\right)=1$ )?

The paper is organized as follows: $\$ 1$ and $\$ 2$ establish basic facts about pmp groupoids and their actions. In the case of $s(G)$, the correspondence "groupoids $\leftrightarrow$ equivalence relations" is achieved by using the formula $s(G)=s\left(G \ltimes X_{0}^{G}\right)$, where $G \curvearrowright X_{0}^{G}$ is a Bernoulli shift, see Theorem 28 in $\$ 7$ (other applications of the correspondence principle are given in 99). The proof of this formula uses the idea in a result of L. Bowen [Bow, Theorem 8.1] for the sofic entropy, as explained in 84. Other difficulties inherent to the groupoid setting are dealt with in $\$ 3$, 95 (these difficulties were avoided in [DKP2] by working with groups and their actions rather than with general groupoids). The proof of Theorem 28 is given in Section 7. In $\$ 8$ we prove a scaling formula for $s(G)$. In $\$ 9$ we prove Theorem 1 by putting together these ingredients.

\section{REview of $s(G)$}

Recall that a discrete standard Borel groupoid $G$ with base (=set of objects) $G^{0}$, source map s: $G \rightarrow G^{0}$ and range map r: $G \rightarrow G^{0}$, is said to be probability measure preserving (pmp) with respect to a Borel probability measure $\mu$ on $G^{0}$ if the left and right Haar measures $\mathfrak{h}$ and $\mathfrak{h}^{-1}$ on $G$ coincide:

$$
\mathfrak{h}=\mathfrak{h}^{-1}
$$

where

$$
\mathfrak{h}(A):=\int_{G^{0}}\left|A^{e}\right| \mathrm{d} \mu(e) \text { and } \mathfrak{h}^{-1}(A):=\int_{G^{0}}\left|A_{e}\right| \mathrm{d} \mu(e)
$$

and $A \subset G$ is a Borel set with $A^{e}:=\mathbf{r}^{-1}(e) \cap A$ and $A_{e}:=\mathbf{s}^{-1}(e) \cap A$ for $e \in G^{0}$. Then $\mathfrak{h}_{\mid G^{0}}=\mathfrak{h}_{\mid G^{0}}^{-1}=\mu$ so we simply denote by $\mathfrak{h}$ the measure $\mu$ on $G^{0}$.

A bisection is a Borel subset $s \subset G$ such that the restrictions of $\mathbf{s}$ and $\mathbf{r}$ to $s$ are Borel isomorphisms onto $G^{0}$. The set of bisections form a group called the full group of $G$ and are denoted $[G]$. A partial bisection is a Borel subset $s \subset G$ such that $\mathbf{s}$ and $\mathbf{r}$ are injective in restriction to $s$. The set of partial bisection form a Polish inverse monoid called the full inverse semigroup (or the full pseudogroup) of $G$ and denoted $\llbracket G \rrbracket$. For $s \in \llbracket G \rrbracket \operatorname{let} \operatorname{dom}(s):=s^{-1} s \subset G^{0}$ and $\operatorname{ran}(s):=s s^{-1} \subset G^{0}$.

For example, if $G:=\{1, \ldots, d\}^{2}$ is the transitive equivalence relation on the set $\{1, \ldots d\}$ with $d \in \mathbb{Z}_{\geq 1}$ elements, then $[G]=\operatorname{Sym}(d)$ is the symmetric group on $d$ letters and $\llbracket G \rrbracket$ is the inverse semigroup of partial permutations. We denote the latter by $\llbracket d \rrbracket$.

The semigroup $\llbracket G \rrbracket$ (and $[G] \subset \llbracket G \rrbracket$ ) is Polish with respect to the uniform distance

$$
|s-t|:=\mathfrak{h}\left\{e \in G^{0} \mid s e \neq t e\right\} .
$$


If $G=\{1, \ldots, d\}^{2}$ then the uniform distance is the normalized Hamming distance on $\llbracket d \rrbracket$.

The (von Neumann) trace on $\llbracket G \rrbracket$ is given by

$$
\tau(s):=\mathfrak{h}\left(s \cap G^{e}\right)=\mathfrak{h}\left\{e \in G^{0} \mid s e=e\right\} .
$$

It is the restriction to $\llbracket G \rrbracket \subset L G$ of the finite trace on the von Neumann algebra $L G$ of $G$.

We have

$$
|s-t|=\tau\left(s^{-1} s\right)+\tau\left(t^{-1} t\right)-\tau\left(s^{-1} s t^{-1} t\right)-\tau\left(s t^{-1}\right) .
$$

We will write $\operatorname{tr}$ for the trace on $\llbracket d \rrbracket$. So

$$
\operatorname{tr}(\sigma)=\frac{1}{d} \times \text { number of fixed points of } \sigma \in \llbracket d \rrbracket \text {. }
$$

If $F \subset \llbracket G \rrbracket$ is a finite subset and $n \in \mathbb{Z}_{\geq 1}$ then $F_{ \pm}^{n}$ denotes the set of all products of at most $n$ elements of $F_{ \pm}:=F \cup F^{-1} \cup\{\operatorname{Id}\}$. For $F \subset \llbracket G \rrbracket$ we let $\boldsymbol{\Sigma} F \subset \llbracket G \rrbracket$ denote the set of sums of elements of $F$ with pairwise orthogonal domains and pairwise orthogonal ranges.

By definition, $G$ is sofic if its full inverse semigroup $\llbracket G \rrbracket$ is sofic:

Definition 3. A pmp groupoid $G$ is called sofic if for every finite set $F \subset \llbracket G \rrbracket, \delta>0$ and $n \in \mathbb{Z}_{\geq 1}$ there exist $d \in \mathbb{Z}_{\geq 1}$ and a map

$$
\sigma: \Sigma F_{ \pm}^{n} \rightarrow \llbracket d \rrbracket
$$

such that

$$
|\sigma(s t)-\sigma(s) \sigma(t)|<\delta
$$

for every $s, t \in \boldsymbol{\Sigma} F_{ \pm}^{n}$ such that $s t \in \boldsymbol{\Sigma} F_{ \pm}^{n}(\sigma$ is $\delta$-multiplicative $)$ and

$$
|\operatorname{tr} \circ \sigma(s)-\tau(s)|<\delta
$$

for every $s \in F_{ \pm}^{n}$ ( $\sigma$ is $\delta$-trace-preserving).

Remark 4. If $G$ is a group, one can replace $\llbracket d \rrbracket$ by $\operatorname{Sym}(d)$ as is easily seen.

Remark 5. The notion of sofic pmp equivalence relations was introduced by Elek and Lippner in [EL] in terms of graph approximation and in $\mathrm{Oz}$ by requiring that $\llbracket G \rrbracket$ is sofic as in the definition above (compare [DKP1, DKP2]). The sofic property was first considered for groups by Gromov and Weiss in terms of (Cayley) graph approximation and was studied by Elek and Szabo. It is a simultaneous generalization of amenability and the LEF property of Vershik and Gordon (see Pe for more details).

Let

and define

$$
\mathrm{SA}(F, n, \delta, d):=\left\{\sigma: \Sigma F_{ \pm}^{n} \rightarrow \llbracket d \rrbracket \text { satisfying (i), (ii) }\right\}
$$

for $E \subset F$.

$$
|\mathrm{SA}(F, n, \delta, d)|_{E}:=\left|\left\{\sigma_{\mid E} \mid \sigma \in \mathrm{SA}(F, n, \delta, d)\right\}\right|
$$

Definition 6. For $E \subset F \subset \llbracket G \rrbracket$ finite, $n \in \mathbb{Z}_{\geq 1}$ and $\delta>0$ define successively

$$
\begin{aligned}
s_{E}(F, n, \delta) & :=\limsup _{d \rightarrow \infty} \frac{\log |\mathrm{SA}(F, n, \delta, d)|_{E}}{d \log d} \\
s_{E}(F, n) & :=\inf _{\delta>0} s_{E}(F, n, \delta) \\
s_{E}(F) & :=\inf _{n \in \mathbb{Z}_{\geq 1}} s_{E}(F, n)
\end{aligned}
$$


If $K \subset \llbracket G \rrbracket$ is an arbitrary subset the sofic dimension of $K$ is

$$
s(K):=\sup _{E} \inf _{F} s_{E}(F)
$$

where $E \subset F \subset K$ are finite subsets. The sofic dimension of $G$ is $s(G):=s(\llbracket G \rrbracket)$. One defines similarly the lower sofic dimension $\underline{s}$ and the $\omega$ sofic dimension $s^{\omega}$ for a ultrafilter $\omega$ on $\mathbb{Z}_{\geq 1}$ by replacing $\limsup _{d \rightarrow \infty}$ by $\liminf _{d \rightarrow \infty}$ and $\lim _{d \rightarrow \omega}$ respectively.

Voiculescu's regularity condition reads:

Definition 7. A pmp groupoid $G$ is s-regular if $\underline{s}(G)=s(G)$.

Finally we recall

Definition 8. A subset $K \subset \llbracket G \rrbracket$ is transversally generating if for any $t \in \llbracket G \rrbracket$ and $\varepsilon>0$ there exist $n \in \mathbb{Z}_{\geq 1}$ and $s \in K_{ \pm}^{n}$ such that $|t-s| \leq \varepsilon$.

This definition appears in [DKP1, Definition 2.4] where it is called "dynamically generating". The more classical notion of generating set for pmp equivalence relations (and groupoids) (as in [DKP1, Definition 2.2]) is that of ConnesFeldmann-Weiss. While being distinct notions, a groupoid is finitely generated in the Connes-Feldmann-Weiss sense if and only if it is transversally finitely generated (by an argument similar to that in [DKP1, Proposition 2.6]) so 'finitely generated' is unambiguous for groupoids (and coincide with the usual notion in the group case).

The following result is proved in [DKP1, Theorem 4.1].

Theorem 9 (Invariant of $s$ under orbit equivalence). Let $R$ be pmp equivalence relation and $K, L$ be transversally generating sets. Then $s(K)=s(L), \underline{s}(K)=\underline{s}(L)$ and $s^{\omega}(K)=s^{\omega}(L)$.

The result in [DKP1] is stated for finitely generated equivalence relations but the same proof works in the general case (as does the proof of [DKP1, Theorem 1.2]). The proof in full generality for groupoids is given in [DKP2, Theorem 2.11]. We won't use this more general result here but will rather deduce it from Theorem 9 as an illustration of the correspondence principle.

Remark 10. It is sometimes convenient to use the 2-norm on $L G$ and its restriction to $\llbracket G \rrbracket$ :

$$
\|s-t\|_{2}^{2}:=\tau\left((s-t)(s-t)^{-1}\right)=\tau\left((s-t)^{-1}(s-t)\right)
$$

Observe

$$
\|s-t\|_{2}^{2} \geq|s-t|
$$

(with equality on $[G] \subset \llbracket G \rrbracket$ ) as

$$
\|s-t\|_{2}^{2}=\tau\left(s^{-1} s\right)+\tau\left(t^{-1} t\right)-2 \tau\left(s t^{-1}\right)
$$

and $\tau\left(s t^{-1}\right) \leq \tau\left(s^{-1} s t^{-1} t\right)$.

\section{ACtions of GROUPOIDS}

Let $X$ be a standard Borel space endowed with a Borel fibration $p: X \rightarrow G^{0}$ where $G^{0}$ is the base of $G$. If $\left(\mu^{e}\right)_{e \in G^{0}}$ is a Borel field of probability measures on $X$ we define a probability measure $\mu$ on $X$ by $\mu:=\int_{G^{0}} \mu^{e} \mathrm{~d} \mathfrak{h}(e)$ where $\mathfrak{h}$ is the invariant 
Haar measure on $G^{0}$. Recall that a pmp action of $G$ on the fibered space $(X, \mu)$ is a measurable map

$$
G *_{G^{0}} X \ni(g, x) \mapsto g x \in X
$$

(where $G$ fibers via the source map s : $G \rightarrow X_{G}$ ) satisfying the usual axioms of an action, and such that

$$
g_{*} \mu^{\mathbf{s}(g)}=\mu^{\mathbf{r}(g)}
$$

for ae $g \in G$. Groupoid actions are denoted $G \curvearrowright X$. The crossed product groupoid is the fiber bundle $G \times_{G^{0}} X$ endowed with groupoid law defined by $(s, x)(t, y)=(s t, y)$ whenever $t(y)=x$.

Example 11 (Bernoulli shifts). Given a pmp groupoid $G$ with invariant Haar measure $\mathfrak{h}$ and a probability space $\left(X_{0}, \mu_{0}\right)$, consider the probability space

$$
\left(X_{0}^{G}, \mu\right):=\int_{G^{0}}\left(X_{0}^{G^{e}}, \mu_{0}^{\otimes G^{e}}\right) \mathrm{d} \mathfrak{h}(e),
$$

(where $X_{0}^{G^{e}}:=\prod_{G^{e}} X_{0}$ is the infinite Cartesian product over $G^{e}:=\mathbf{r}^{-1}(e)$ ), endowed with the fibration $X_{0}^{G} \rightarrow G^{0}$ and field of measures $\left(\mu_{0}^{\otimes G^{e}}\right)_{e \in G^{0}}$. Every element $x \in X_{0}^{G}$ can be viewed as a sequence $x:=\left(x_{t}\right)_{t \in G^{e}}$ of elements of $X_{0}$. The Bernoulli action $G \curvearrowright X_{0}^{G}$ is given by

$$
s\left(\left(x_{t}\right)_{t \in G^{\mathrm{r}(s)}}\right):=\left(\left(x_{s^{-1} t}\right)_{t \in G^{\mathrm{r}(s)}}\right) .
$$

Remark 12. The notion of groupoid action has long been used in ergodic group theory (see for example $[\mathrm{Ram}]$ ). They can equivalently be described as actions on bundles (cocycles) as above, or as groupoid extensions which are fiber bijective. In Bow2 L. Bowen discusses Bernoulli shifts using the latter description.

We first prove a few lemmas that will be used in the proof of Theorem 1

Lemma 13. Let $G \curvearrowright X_{0}^{G}$ be a Bernoulli action and $H \subset G$ be an ergodic subgroupoid with $G^{0}=H^{0}$. Then the action $H \curvearrowright X_{0}^{G}$ is isomorphic to a Bernoulli shift over $H$.

Proof. By the von Neumann selection theorem, we can find a measurable section $s: R G \rightarrow G$ of $(\mathbf{s}, \mathbf{r}): G \rightrightarrows R G$ (=the pmp equivalence relation associated with $G$ ) such that that $s\left(R G^{0}\right)=G^{0}$ and $s(R H) \subset H$ and, since the set $G(e) / H(e)$ is countable for $e \in G^{0}$, measurable sections $\left(g_{j}: D_{j} \subset G^{0} \rightarrow S G\right)_{j \in J}$ of $S G \rightarrow G^{0}$ such that for ae $e \in G^{0}, G(e)=\bigsqcup_{j \in J} H(e) g_{j}(e)$. By ergodicity we may assume $\mathfrak{h}\left(D_{j}\right)=1$. Let $\left(\varphi_{i}\right)_{i \in I}$ be a sequence in $\operatorname{Aut}\left(G^{0}\right)$ such that $\left\{R H\left[\varphi_{i} e\right]\right\}_{i \in I}$ form a partition of $R G[e]$ for ae $e \in G^{0}$ (see [FSZ]). Then

$$
G^{e}=\bigsqcup_{i \in I, j \in J} \bigsqcup_{(e, f) \in R H} H(e) g_{j}(e) s\left(e, \varphi_{i}^{-1} f\right)
$$

since any $g \in G^{e}$ can be written uniquely in the form $h_{0} g_{j}(e) s\left(e, \varphi_{i}^{-1} f\right)$ for $i$ and $f$ such that $(e, f) \in R H$ and $f=\varphi_{i} \mathbf{s}(g)$ so $g s\left(e, \varphi_{i}^{-1} f\right)^{-1} \in G(e)$ and $h_{0}=$ $g s\left(e, \varphi_{i}^{-1} f\right)^{-1} g_{j}(e)^{-1} \in H(e)$.

Consider the measurable field of maps $\psi_{e}: X_{0}^{G^{e}} \rightarrow\left(X_{0}^{I \times J}\right)^{H^{e}}$ defined by sending $x \in X_{0}^{G^{e}}$ to

$$
\left(\left(x_{h_{0} g_{j}(e) s\left(e, \varphi_{i}^{-1} f\right)}\right)_{(i, j) \in I \times J}\right)_{h_{0} \in H(e),(e, f) \in R H} .
$$


These maps are measure preserving and if we consider the Bernoulli action of $H$ with base $X_{0}^{I \times J}$ then we see it is $H$-equivariant: for $h \in H^{e}$ say $h=h_{1}^{-1} s(d, e)^{-1}$ where $h_{1} \in H(e)$

$$
\begin{aligned}
\psi_{d}(h(x)) & =\psi\left(h\left(x_{h_{0} g_{j}(e) s\left(e, \varphi_{i}^{-1} f\right)}\right)_{(i, j) \in I \times J, h_{0} \in H(e),(e, f) \in R H}\right) \\
& =\psi\left(\left(x_{s(d, e) h_{1} h_{0} g_{j}(e) s\left(e, \varphi_{i}^{-1} f\right)}\right)_{(i, j) \in I \times J, h_{0} \in H(e),(e, f) \in R H}\right) \\
& =\left(\left(x_{s(d, e) h_{1} h_{0} g_{j}(e) s\left(e, \varphi_{i}^{-1} f\right)}\right)_{(i, j) \in I \times J}\right)_{h_{0} \in H(e),(e, f) \in R H} \\
& =h\left(\left(x_{h_{0} g_{j}(e) s\left(e, \varphi_{i}^{-1} f\right)}\right)_{(i, j) \in I \times J}\right)_{h_{0} \in H(e),(e, f) \in R H} \\
& =h\left(\psi_{e}(x)\right)
\end{aligned}
$$

We say a groupoid action $G \curvearrowright X$ is essentially free if for ae $s \in G \backslash G^{0}$

$$
\mu^{\mathbf{s}(s)}(\operatorname{Fix}(s))=0
$$

where

$$
\operatorname{Fix}(s)=\left\{x \in X^{\mathbf{s}(s)} \mid s x=x\right\} .
$$

Lemma 14. If the pmp groupoid action $G \curvearrowright X$ is essentially free then $G \ltimes X$ is a pmp equivalence relation.

Proof. Since $G \curvearrowright X$ is essentially free and $G_{e}$ is countable the set

$$
X_{0}^{e}:=\left\{x \in X^{e} \mid s x \neq x, \forall s \in G_{e}, s \neq e\right\}
$$

has measure 1 in $X^{e}$ for every $e \in A \subset G^{0}$ a measurable subset with $\mathfrak{h}(A)=1$. Let $\left(X_{0}^{A}, \mu\right) \rightarrow(A, \mathfrak{h})$ be the measure fibration corresponding to $\left(X_{0}^{e}\right)_{e \in A}$. Since $G$ is isomorphic to $G_{\mid A} \ltimes X_{0}^{A}$, we may assume that $s x \neq x$ for all $s \in G \backslash G^{0}, x \in X^{\mathbf{s}(s)}$. Then for $(s, x) \in G \ltimes X$

$$
\mathbf{r}(s, x)=\mathbf{s}(s, x) \Leftrightarrow x \in \operatorname{Fix}(s)
$$

so $\mathbf{r}(s, x) \neq \mathbf{s}(s, x)$ for every $s \in G \backslash G^{0}$ and $x \in X$. This shows that $G \ltimes X$ is an equivalence relation. It is an easy exercise to check that it is pmp (more generally if $G$ is pmp and $G \curvearrowright X$ is a pmp action, then $G \ltimes X$ is a pmp groupoid).

Lemma 15. If $G$ is transversally finitely generated then so is $G \ltimes X$ for any ergodic pmp action $G \curvearrowright X$.

Proof. Let $R$ be the orbit equivalence relation of $G \curvearrowright X$. Since $R$ is ergodic, there exists an ergodic automorphism $\theta \in \llbracket R \rrbracket$, which is orbit equivalent to a Bernoulli shift $\mathbb{Z} \curvearrowright\{0,1\}^{\mathbb{Z}}(\overline{\text { Dye }})$. For $i \in\{0,1\}$ let $B_{i}$ be the cylinder set $\left\{x \in\{0,1\}^{\mathbb{Z}} \mid x_{0}=i\right\}$. Let $p_{1}, p_{2}$ be the projection in $L^{\infty}(X)$ corresponding to $B_{0}$ and $B_{1}$ in $\mathbb{Z} \curvearrowright\{0,1\}^{\mathbb{Z}}$. If $F \subset \llbracket G \rrbracket$ be a finite transversally generating set for $G$, then $F \cup\left\{\theta, p_{1}, p_{2}\right\}$ is a finite generating set for $G \ltimes X$.

Lemma 16. The Bernoulli action $G \curvearrowright X_{0}^{G}$ is essentially free if $G$ has infinite fibers (i.e. $\left|G^{e}\right|=\infty$ for ae $e \in G^{0}$ ), and the support of $\mu_{0}$ contains at least two points. If in addition $\mu_{0}$ is diffuse then the action is essentially free.

Proof. Let $s \in G^{e}, s \neq e$, such that $\left|G^{e}\right|=\infty$. We show that $\mu^{e}(\operatorname{Fix}(s))=0$. If $\mathbf{r}(s) \neq \mathbf{s}(s)$ this is clear so we assume $s \in G(e)$. Note that $\left(x_{t}\right)_{t \in G e}$ is $\langle s\rangle$-invariant if and only if $x_{s^{n} t}=x_{t}$ for all $n \in \mathbb{Z}, t \in G^{e}$. Thus we can find an infinite family of pairwise disjoint pairs $\left\{s_{i}, t_{i}\right\}_{i \in I}, s_{i} \neq t_{i} \in G^{e}$, such that $x_{s_{i}}=x_{t_{i}}$ for every $x \in \operatorname{Fix}(s)$ 
and $i \in I$. Since $|I|=\infty$, the set of $x \in X_{0}^{G^{e}}$ such that $x_{s_{i}}=x_{t_{i}}$ is negligible, so $\mu^{e}(\operatorname{Fix}(s))=0$. If in addition $\mu_{0}$ is diffuse then the set of $x \in X_{0}^{G^{e}}$ such that $x_{s}=x_{e}$ is negligible so $\mu^{e}(\operatorname{Fix}(s))=0$ in this case.

Lemma 17. If $G$ is ergodic with infinite fibers then the Bernoulli action $G \curvearrowright X_{0}^{G}$ is ergodic for any base space $\left(X_{0}, \mu_{0}\right)$.

Proof. If $A \subset X_{0}^{G}$ is a nonzero $G$-invariant subset and $p: X_{0}^{G} \rightarrow G^{0}$, then $p(A)$ is $G$-invariant and nonzero therefore $\delta:=\mu^{e}\left(A^{e}\right) \neq 0$ and is almost surely constant by the ergodicity of $G$. Let $\varepsilon>0$ be arbitrary. Since $\left|G^{e}\right|=\infty$ almost surely we can find by the von Neumann selection theorem a nonzero measure field $\left(F^{e}\right)_{e \in B}$ of finite subsets of $\left(G^{e}\right)_{e \in B}$ such that $\mu^{e}\left(A^{e} \cap A_{\mid F^{e}}\right)>\delta-\varepsilon$ and a section $s$ of $\mathbf{s}$ such that $s_{e} F^{e} \cap F^{\mathbf{r}\left(s_{e}\right)}=\varnothing$ for ae $e \in B$. By invariance $\mu^{\mathbf{r}(e)}\left(A^{\mathbf{r}\left(s_{e}\right)} \cap A_{\mid s_{e} F^{e}}\right)>\delta-\varepsilon$ so

$$
\mu^{\mathbf{r}(e)}\left(A_{\mid F^{\mathbf{r}\left(s_{e}\right)}} \cap A_{\mid s_{e} F^{e}}\right)>\delta-2 \varepsilon
$$

and $\mu^{\mathbf{r}(e)}\left(A_{\mid F^{\mathbf{r}\left(s_{e}\right)}} \cap A_{\mid s_{e} F^{e}}\right)=\mu^{\mathbf{r}(e)}\left(A_{\mid F^{\mathbf{r}\left(s_{e}\right)}}\right) \mu^{\mathbf{r}(e)}\left(A_{\mid s_{e} F^{e}}\right)$ also

$$
\mu^{\mathbf{r}(e)}\left(A_{\mid F^{\mathbf{r}\left(s_{e}\right)}} \cap A_{\mid s_{e} F^{e}}\right)<\delta^{2}+6 \varepsilon \delta+2 \varepsilon^{2} .
$$

Letting $\varepsilon \rightarrow 0$, we get $\delta^{2} \geq \delta$ so $\delta \geq 1$ and $\mu(A)=1$.

Lemma 18. Let $G \curvearrowright X$ be a groupoid action and suppose that $G=G_{1} *_{G_{3}} G_{2}$. Then

$$
G \ltimes X \simeq\left(G_{1} \ltimes X_{\mid 1}\right){ }_{\left(G_{3} \ltimes X_{\mid 3}\right)}\left(G_{2} \ltimes X_{\mid 2}\right)
$$

where $X_{\mid i}=p^{-1}\left(G_{i}^{0}\right)$.

Proof. First note that $G_{i}$ acts on $X_{i}$ and that $G_{i} \ltimes X_{\mid i}$ is naturally a subgroupoid of $G \ltimes X$. Given an arbitrary groupoid $H$ and groupoid morphisms $f_{1}: G_{1} \ltimes X_{\mid 1} \rightarrow H$, $f_{2}: G_{2} \ltimes X_{\mid 2} \rightarrow H$ with $\left.f_{1}\right|_{G_{3} \ltimes X_{3}}=\left.f_{2}\right|_{G_{3} \ltimes X_{\mid 3}}$ we want to show there is a unique morphism $k: G \ltimes X \rightarrow H$ such that the following diagram commutes (where the unlabeled edges are the inclusion map).

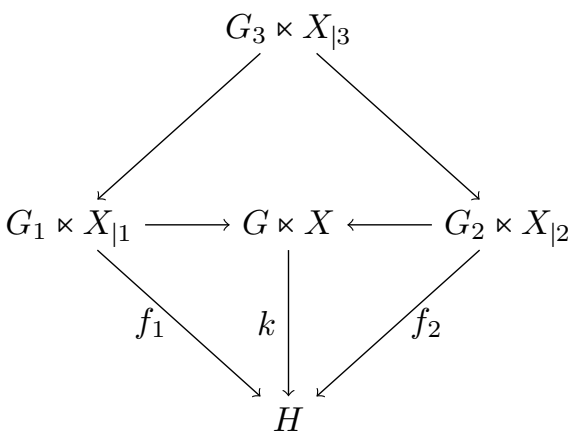

Since $\left.k\right|_{G_{1} \ltimes X_{11}}=\left.f_{1}\right|_{G_{1} \ltimes X_{\mid 1}},\left.k\right|_{G_{2} \ltimes X_{\mid 2}}=\left.f_{2}\right|_{G_{2} \ltimes X_{\mid 2}}$ and the values of $k$ are determined on $G_{1} \ltimes X_{\mid 1}$ and $G_{2} \ltimes X_{\mid 2}$ which generate $G \ltimes X$, this gives uniqueness. To show it is well defined note that if $g_{i} \in G_{1} \ltimes X^{G_{1}} \cup G_{2} \ltimes X^{G_{2}}$ and $g_{1} \ldots g_{n}=\operatorname{Id}_{\mathbf{s}\left(g_{1}\right)}$ and if $g_{i}^{\prime}$ are the corresponding elements of $G_{1}$ and $G_{2}$ then $g_{1}^{\prime} \ldots g_{n}^{\prime}=e$, so using the fact that $G_{1}$ and $G_{2}$ are in free product over $G_{3}$ this shows that $k\left(g_{1}\right) \ldots k\left(g_{n}\right)=\operatorname{Id}_{\mathbf{s}\left(f_{1}\left(g_{1}\right)\right)}$ or $\operatorname{Id}_{\mathbf{s}\left(f_{2}\left(g_{1}\right)\right)}$ as appropriate. 


\section{OvERLAPPING GENERATORS}

Let $F \subset \llbracket G \rrbracket$ be a finite subset, $\pi$ be a partition of $F$, and $\sigma: F \rightarrow \llbracket d \rrbracket$ be a map. Denote $F_{\mid \pi}$ the set of $e \in G^{0}$ such that

$$
e \leq \prod_{s \in F} s s^{-1}
$$

and

$$
\forall s, t \in F, e s=e t \Longleftrightarrow \pi(s)=\pi(t) .
$$

Here and below we will view a partition $\pi$ of $F$ as a map from $F$ to $\{1, \ldots, \alpha\}=: \operatorname{ran} \pi$ for some $\alpha \in \mathbb{Z}_{\geq 0}$.

Similarly let $F_{\mid \pi}^{\sigma}$ be the set of $e \in\{1, \ldots d\}$ (identified with the base space of the transitive relation on $\{1, \ldots, d\})$ such that

$$
e \leq \prod_{s \in F} \sigma(s) \sigma(s)^{-1}
$$

and

$$
\forall s, t \in F, e \sigma(s)=e \sigma(t) \Longleftrightarrow \pi(s)=\pi(t) .
$$

The following lemma will be useful in the proof of Theorem 28 . We denote a projection onto a set $A$ by $p_{A}$.

Lemma 19. Given $F \subset \llbracket G \rrbracket, n \geq 1$, let

$$
F_{n}:=F \cup\left\{p_{F_{0 \mid \pi}} \mid F_{0} \subset F_{ \pm}^{n}, \pi \text { partition of } F_{0}\right\}
$$

If $\sigma \in \mathrm{SA}\left(F_{n}, 4 n\left|F_{ \pm}^{n}\right|+1, \delta, d\right), F_{0} \subset F_{ \pm}^{n}$, and $\pi$ is a partition of $F_{0}$ then

$$
\left|\mathfrak{h}\left(F_{0 \mid \pi}\right)-\frac{\left|F_{0 \mid \pi}^{\sigma}\right|}{d}\right|<c_{1}(F, n) \delta
$$

where $c_{1}(F, n)=176\left(3^{2 n}\right)\left|F_{ \pm}\right|^{2 n}$.

Proof. Fix some $F_{0} \subset F_{ \pm}^{n}$ and let $\pi_{1}, \pi_{2}, \ldots, \pi_{m}$ be the family of partitions of $F_{0}$ (so $m=\mathrm{Be}\left(\left|F_{0}\right|\right)$ is the Bell number of $\left|F_{0}\right|$ ). For convenience let $p_{j}:=p_{F_{0 \mid \pi_{j}}}$ and $p_{j}^{\sigma}:=p_{F_{0 \mid \pi_{j}}^{\sigma}}$.

$$
\begin{aligned}
& \text { If } \pi_{j}(s)=\pi_{j}(t), p_{j}=s t^{-1} p_{j} \text { so } \\
& \qquad \begin{aligned}
&\left|\sigma(s) \sigma(t)^{-1} \sigma\left(p_{j}\right)-\sigma\left(p_{j}\right)\right|=\left|\sigma(s) \sigma(t)^{-1} \sigma\left(p_{j}\right)-\sigma\left(s t^{-1} p_{j}\right)\right| \\
& \leq\left|\sigma(s) \sigma(t)^{-1} \sigma\left(p_{j}\right)-\sigma(s) \sigma\left(t^{-1}\right) \sigma\left(p_{j}\right)\right|+\left|\sigma(s) \sigma\left(t^{-1}\right) \sigma\left(p_{j}\right)-\sigma\left(s t^{-1} p_{j}\right)\right| \\
& \quad \leq 4 \delta+3 \delta=7 \delta
\end{aligned}
\end{aligned}
$$

by [DKP1, Lemma 3.1(8)].

Similarly if $\pi_{j}(s) \neq \pi_{j}(t)$ then

$$
\left|\sigma(s) \sigma(t)^{-1} \sigma\left(p_{j}\right)-\sigma\left(s t^{-1} p_{j}\right)\right| \leq 7 \delta .
$$

As $\tau\left(s t^{-1} p_{j}\right)=0$ we have

$$
\begin{aligned}
& \left|\operatorname{tr}\left(\sigma(s) \sigma(t)^{-1} \sigma\left(p_{j}\right)\right)\right|=\left|\operatorname{tr}\left(\sigma(s) \sigma(t)^{-1} \sigma\left(p_{j}\right)\right)-\tau\left(s t^{-1} p_{j}\right)\right| \\
& \leq\left|\operatorname{tr}\left(\sigma(s) \sigma(t)^{-1} \sigma\left(p_{j}\right)\right)-\operatorname{tr} \circ \sigma\left(s t^{-1} p_{j}\right)\right|+\left|\operatorname{tr} \circ \sigma\left(s t^{-1} p_{j}\right)-\tau\left(s t^{-1} p_{j}\right)\right| \\
& \quad \leq 7 \delta+\delta=8 \delta
\end{aligned}
$$

by [DKP1, Lemma 3.1(4)] 
Moreover

$$
\left|\sigma\left(p_{j}\right)-\sigma\left(p_{j}\right) \sigma\left(p_{j}\right)\right|=\left|\sigma\left(p_{j}^{2}\right)-\sigma\left(p_{j}\right) \sigma\left(p_{j}\right)\right|<\delta
$$

and since $\sum_{j=1}^{m} p_{j}=\prod_{s \in F_{0}} s s^{-1}$ using [DKP1, Lemma 3.5]

$$
\begin{aligned}
\left|\sum_{j=1}^{m} \sigma\left(p_{j}\right)-\prod_{s \in F_{0}} \sigma(s) \sigma(s)^{-1}\right| & <\left|\sigma\left(\prod_{s \in F_{0}} s s^{-1}\right)-\prod_{s \in F_{0}} \sigma(s) \sigma(s)^{-1}\right|+150(2|F|+1)^{2 n} \delta \\
& \leq\left|F_{0}\right| 4 \delta+\left|F_{0}\right| 2 \delta+\delta+150(2|F|+1)^{2 n} \delta \\
& \leq 157\left(3^{2 n}\right)\left|F_{ \pm}\right|^{2 n} \delta .
\end{aligned}
$$

If $V$ denotes the set of integers $1 \leq k \leq d$ such that for all $1 \leq j \leq m$ and $s, t \in F_{0}$ :

$$
\begin{gathered}
\sigma(s) \sigma(t)^{-1} \sigma\left(p_{j}\right) k=\sigma\left(p_{j}\right) k \\
\sigma(s) \sigma(t)^{-1} \sigma\left(p_{j}\right) k \neq k \\
\sigma\left(p_{j}\right) k=\sigma\left(p_{j}\right) \sigma\left(p_{j}\right) k \\
\left(\sum_{j=1}^{m} \sigma\left(p_{j}\right)\right) k=\left(\prod_{s \in F_{0}} \sigma(s) \sigma(s)^{-1}\right) k
\end{gathered}
$$

then

$$
\begin{aligned}
|V| & \geq\left(1-7 \delta\left|F_{ \pm}^{n}\right|-7 \delta\left|F_{ \pm}^{n}\right|-\delta\left|F_{ \pm}^{n}\right|-\delta\left|F_{ \pm}^{n}\right|-157\left(3^{2 n}\right)\left|F_{ \pm}\right|^{2 n} \delta\right) d \\
& \geq\left(1-175\left(3^{2 n}\right)\left|F_{ \pm}\right|^{2 n} \delta\right) d
\end{aligned}
$$

So, for all $\pi_{j}$ we have that $\sigma\left(p_{j}\right)_{\mid V}$ is a projection and

$$
\pi_{j}(s)=\pi_{j}(t) \Rightarrow \sigma(s) \sigma(t)^{-1} \sigma\left(p_{j}\right)_{\mid V}=\sigma\left(p_{j}\right)_{\mid V} \text { and } \operatorname{tr}\left(\sigma(s) \sigma(t)^{-1} \sigma\left(p_{j}\right)_{\mid V}\right)=0
$$

Hence $\operatorname{ran}\left(\sigma\left(p_{j}\right) p_{V}\right) \subset \operatorname{ran}\left(p_{j}^{\sigma} p_{V}\right)$, but since we also have that

$$
\left(\sum_{j=1}^{m} \sigma\left(p_{j}\right)\right) p_{V}=\left(\prod_{s \in F_{0}} \sigma(s) \sigma(s)^{-1}\right) p_{V}=\left(\sum_{j=1}^{m} p_{j}^{\sigma}\right) p_{V}
$$

we conclude $\sigma\left(p_{j}\right) p_{V}=p_{j}^{\sigma} p_{V}$ which implies $\left|\operatorname{tr} \circ \sigma\left(p_{j}\right)-\right| F_{0 \mid \pi_{j}}^{\sigma}|/ d|<175\left(3^{2 n}\right)\left|F_{ \pm}\right|^{2 n} \delta$ therefore

$$
\begin{aligned}
\left|\mathfrak{h}\left(F_{0}^{\pi_{j}}\right)-\right| F_{0 \mid \pi_{j}}^{\sigma}|/ d| & =\left|\operatorname{tr}\left(p_{j}\right)-\right| F_{0 \mid \pi_{j}}^{\sigma}|/ d| \\
& \leq\left|\operatorname{tr}\left(p_{j}\right)-\operatorname{tr} \circ \sigma\left(p_{j}\right)\right|+\left|\operatorname{tr} \circ \sigma\left(p_{j}\right)-\right| F_{0 \mid \pi_{j}}^{\sigma}|/ d| \\
& \leq \delta+175\left(3^{2 n}\right)\left|F_{ \pm}\right|^{2 n} \delta \leq 176\left(3^{2 n}\right)\left|F_{ \pm}\right|^{2 n} \delta .
\end{aligned}
$$

\section{BERNOUlli SHIFTS AND RANDOM PARTITIONS}

For $1 \leq i \leq q$ let

$$
B_{i}:=\left\{x \in\{1, \ldots, q\}^{G} \mid x_{e}=i\right\}
$$

where $X=\{1, \ldots, q\}^{G}$ is endowed with the Bernoulli action $G \curvearrowright X$, and for $d \in \mathbb{Z}_{\geq 1}$ let $A_{1}, \ldots, A_{q}$ be a partition of $\{1, \ldots, d\}$. Given a set $F \subset \llbracket G \rrbracket$ and function $\psi: F \rightarrow\{1, \ldots, q\}$ we define

$$
B_{\psi}:=\bigcap_{s \in F} s B_{\psi(s)}
$$


and

$$
A_{\psi}:=\bigcap_{s \in F_{\psi}} \sigma(s) A_{\psi(s)}
$$

where $\sigma: F \rightarrow \llbracket d \rrbracket$.

The next lemma adapts the proof of Theorem 8.1 from L. Bowen's paper [Bow].

Lemma 20. If $d$ is large enough (depending on $F, n$ and $\delta$ ) then there is a partition $\left\{A_{1}, A_{2}, \ldots, A_{q}\right\}$ of $\{1 \ldots d\}$ such that if $\sigma \in \mathrm{SA}\left(F_{n}, 4 n\left|F_{ \pm}^{n}\right|+1, \delta, d\right)$ then for every subset $F_{\psi} \subset F_{ \pm}^{n}$ and every function $\psi: F_{\psi} \rightarrow\{1, \ldots, q\}$

$$
\left|\mu\left(B_{\psi}\right)-\frac{\left|A_{\psi}\right|}{d}\right|<c_{2}(F, n) \delta
$$

where $c_{2}(F, n)=2 c_{1}(F, n) \operatorname{Be}\left(\left|F_{ \pm}\right|^{n}\right)$ and $\mathrm{Be}$ is the Bell number.

Proof. Fix $F_{\psi} \subset F_{ \pm}^{n}$ and $\psi: F_{\psi} \rightarrow\{1, \ldots, q\}$. Create a random partition

$$
\left\{A_{1}, A_{2}, \ldots, A_{q}\right\}
$$

of $\{1 \ldots d\}$ using the following scheme: for each $k \in\{1, \ldots, d\}$ place $k$ in $A_{i}$ with probability $\mu_{0}(i)$.

We will find the probability that $\left|\mu\left(B_{\psi}\right)-\right| A_{\psi}|/ d|<c_{2}(F, n) \delta$.

Let $\pi_{1}, \pi_{2}, \ldots, \pi_{m}$ be the family of partitions of $F_{\psi}$. Let $s_{j}: \operatorname{ran} \pi_{j} \rightarrow F_{\psi}$ be an arbitrary section of $\pi_{j}$. If

$$
\chi_{j}:= \begin{cases}0 & \text { if } \exists s, t \in F_{\psi} \text { such that } \pi_{j}(s)=\pi_{j}(t) \text { and } \psi(s) \neq \psi(t) \\ 1 & \text { otherwise }\end{cases}
$$

then

$$
\begin{aligned}
\mu\left(B_{\psi}\right) & =\mu\left(\bigcap_{s \in F_{\psi}} s B_{\psi(s)}\right) \\
& =\sum_{j=1}^{m} \chi_{j} \mathfrak{h}\left(F_{\psi \mid \pi_{j}}\right) \prod_{r \in \operatorname{ran} \pi_{j}} \mu_{0}\left(\psi\left(s_{j}(r)\right)\right)
\end{aligned}
$$

Since for $\sigma \in \operatorname{SA}\left(F_{n}, 4 n\left|F_{ \pm}^{n}\right|+1, \delta, d\right)$, we have

$$
\frac{\left|A_{\psi}\right|}{d}=\sum_{j=1}^{m} \frac{\left|A_{\psi} \cap F_{\psi \mid \pi_{j}}^{\sigma}\right|}{d}
$$

it will suffice to show by the triangle inequality that for all $j$

$$
\left|\chi_{j} \mathfrak{h}\left(F_{\psi \mid \pi_{j}}\right) \prod_{k \in \operatorname{ran} \pi_{j}} \mu_{0}\left(\psi\left(s_{j}(k)\right)\right)-\frac{\left|A_{\psi} \cap F_{\psi \mid \pi_{j}}^{\sigma}\right|}{d}\right|<2 c_{1}(F, n) \delta .
$$

First suppose $\chi_{j}=0$, so for some $s, t \in F_{\psi}, \pi_{j}(s)=\pi_{j}(t)$ and $\psi(s) \neq \psi(t)$. Then $A_{\psi} \cap F_{\psi \mid \pi_{j}}^{\sigma}=\varnothing$, for if $k \in A_{\psi} \cap F_{\psi \mid \pi_{j}}^{\sigma}$ then $k \in \sigma(s) A_{\psi(s)} \cap \sigma(t) A_{\psi(t)} \cap F_{\psi \mid \pi_{j}}^{\sigma}$ which implies $\sigma(s)^{-1} k=\sigma(t)^{-1} k \in A_{\psi(s)} \cap A_{\psi(t)}$, a contradiction.

Next suppose $\chi_{j}=1$. For $1 \leq k \leq d$ let

$$
Z_{k}= \begin{cases}1 & \text { if } k \in F_{\psi \mid \pi_{j}}^{\sigma} \cap A_{\psi} \\ 0 & \text { otherwise }\end{cases}
$$

We wish to compute $\mathbb{E}\left(Z_{k}\right)$. 
For $k \notin F_{\psi \mid \pi_{j}}^{\sigma}$ we have $\mathbb{E}\left(Z_{k}\right)=0$. Otherwise, $k \in F_{\psi \mid \pi_{j}}^{\sigma} \cap A_{\psi}$ if and only if $\sigma_{s_{j}(r)}^{-1} k \in A_{\psi\left(s_{j}(r)\right)}$ for all $r \in \operatorname{ran} \pi_{j}$ and since

$$
\sigma_{s_{j}(r)}^{-1} k \neq \sigma_{s_{j}\left(r^{\prime}\right)}^{-1} k, \quad \forall r \neq r^{\prime} \in \operatorname{ran} \pi_{j}
$$

we obtain

$$
\mathbb{E}\left(Z_{k}\right)=\prod_{r \in \operatorname{ran} \pi_{j}} \mu_{0}\left(\psi\left(s_{j}(r)\right)\right)
$$

Thus if we let $Z=\sum_{k=1}^{d} Z_{k}=\left|F_{\psi \mid \pi_{j}}^{\sigma} \cap A_{\psi}\right|$ then

$$
\mathbb{E}(Z)=\left|F_{\psi \mid \pi_{j}}^{\sigma}\right| \prod_{r \in \operatorname{ran} \pi_{j}} \mu_{0}\left(\psi\left(s_{j}(r)\right)\right) .
$$

Now lets bound $\operatorname{var}(Z)$ :

$$
\operatorname{var}(Z)=\mathbb{E}\left(Z^{2}\right)-\mathbb{E}(Z)^{2}=\sum_{k, l \in\{1 \ldots, d\}} \mathbb{E}\left(Z_{k} Z_{l}\right)-\mathbb{E}(Z)^{2}
$$

For $k, l \notin F_{\psi \mid \pi_{j}}^{\sigma}$ we have $\mathbb{E}\left(Z_{k} Z_{l}\right)=0=\mathbb{E}\left(Z_{k}\right) \mathbb{E}\left(Z_{l}\right)$. On the other hand if $k, l \in$ $F_{\psi \mid \pi_{j}}^{\sigma}$ then $Z_{k}$ and $Z_{l}$ are not independent if $\sigma_{s_{j}(r)}^{-1} k=\sigma_{s_{j}\left(r^{\prime}\right)}^{-1} l$ for some $r, r^{\prime}$. Thus are at most $\left|F_{ \pm}^{n}\right|^{2}\left|F_{\psi \mid \pi_{j}}^{\sigma}\right|$ non independent pairs $(k, l)$. Now clearly for these pairs $\mathbb{E}\left(Z_{k} Z_{l}\right) \leq \mathbb{E}\left(Z_{k}\right) \mathbb{E}\left(Z_{l}\right)+1$. So returning to our equation above

$$
\begin{gathered}
\sum_{1 \leq k, l \leq d} \mathbb{E}\left(Z_{k} Z_{l}\right)-\mathbb{E}(Z)^{2} \leq \sum_{1 \leq k, l \leq d} \mathbb{E}\left(Z_{k}\right) \mathbb{E}\left(Z_{l}\right)+\left|F_{ \pm}^{n}\right|^{2}\left|F_{\psi \mid \pi_{j}}^{\sigma}\right|-\mathbb{E}\left(Z^{2}\right) \\
=\mathbb{E}\left(Z^{2}\right)+\left|F_{ \pm}^{n}\right|^{2}\left|F_{\psi \mid \pi_{j}}^{\sigma}\right|-\mathbb{E}\left(Z^{2}\right)=\left|F_{ \pm}^{n}\right|^{2}\left|F_{\psi \mid \pi_{j}}^{\sigma}\right|
\end{gathered}
$$

Now we can apply Chebyshev's inequality to $\frac{Z}{d}$ for $a>0$

$$
\operatorname{Pr}\left(\left|\frac{Z}{d}-\frac{\mathbb{E}(Z)}{d}\right| \geq a\right) \leq \frac{\operatorname{var}\left(\frac{Z}{d}\right)}{a^{2}} \leq \frac{\left|F_{ \pm}^{n}\right|^{2}\left|F_{\psi \mid \pi_{j}}^{\sigma}\right|}{a^{2} d^{2}} \leq \frac{\left|F_{ \pm}^{n}\right|^{2}}{a^{2} d}
$$

Since $Z=\left|A_{\psi} \cap F_{\psi \mid \pi_{j}}^{\sigma}\right|$ and $\mathbb{E}(Z)=\left|F_{\psi \mid \pi_{j}}^{\sigma}\right| \prod_{r \in \operatorname{ran} \pi_{j}} \mu_{0}\left(\psi\left(s_{j}(r)\right)\right)$, and, by Lemma 19

$$
\left|\mathfrak{h}\left(F_{\psi \mid \pi_{j}}\right)-\frac{\left|F_{\psi \mid \pi_{j}}^{\sigma}\right|}{d}\right|<c_{1}(F, n) \delta
$$

we have

$$
\operatorname{Pr}\left(\left|\mathfrak{h}\left(F_{\psi \mid \pi_{j}}\right) \prod_{k \in \operatorname{ran} \pi_{j}} \mu_{0}\left(\psi\left(s_{j}(k)\right)\right)-\right| F_{\psi \mid \pi_{j}}^{\sigma} \cap A_{\psi}|/ d| \geq a+c_{1}(F, n) \delta\right) \leq \frac{\left|F_{ \pm}^{n}\right|^{2}}{a^{2} d}
$$

Let $a=c_{1}(F, n) \delta$ so then

$$
\operatorname{Pr}\left(\left|\mathfrak{h}\left(F_{\psi \mid \pi_{j}}\right) \prod_{k \in \operatorname{ran} \pi_{j}} \mu_{0}\left(\psi\left(s_{j}(k)\right)\right)-\right| F_{\psi \mid \pi_{j}}^{\sigma} \cap A_{\psi}|/ d| \geq 2 c_{1}(F, n) \delta\right) \leq \frac{\left|F_{ \pm}^{n}\right|^{2}}{\left(c_{1}(F, n) \delta\right)^{2} d}
$$

So for $d$ large enough there is some partition $\left(A_{1}, \ldots, A_{q}\right)$ such that

$$
\left|\mathfrak{h}\left(F_{\psi \mid \pi_{j}}\right) \prod_{k \in \operatorname{ran} \pi_{j}} \mu_{0}\left(\psi\left(s_{j}(k)\right)\right)-\right| F_{\psi \mid \pi_{j}}^{\sigma} \cap A_{\psi}|/ d|<2 c_{1}(F, n) \delta
$$

Thus, for $d$ large enough there will be a partition so that this true for all $j$ so that

$$
\left|\frac{\left|A_{\psi}\right|}{d}-\mu\left(B_{\psi}\right)\right|<c_{2}(F, n) \delta
$$


Indeed for large enough $d$ nearly all partitions will satisfy this and hence we will have non zero probability that for all $F_{\psi} \subset F_{ \pm}^{n}$ and $\psi: F_{\psi} \rightarrow\{1, \ldots, q\}$ the inequality $\left|\mu\left(B_{\psi}\right)-\right| A_{\psi}|/ d|<c_{2}(F, n) \delta$ holds.

\section{A lemma on apProximate EQUivariance}

Given a groupoid $G$ acting on a set $X$ for any finite set of projections $P$ ᄃ $L^{\infty}(X, \mu)$ and finite set $F \subset \llbracket G \rrbracket$ then $P_{F}$ denotes the set of all projections of the form

$$
\prod_{s \in F^{\prime}} s p_{s}
$$

where $p_{s} \in P$ and $F^{\prime} \subset F$.

Let $P=\left\{p_{B_{i}}\right\}$ where

$$
B_{i}:=\left\{x \in\{1, \ldots, q\}^{G} \mid x_{e}=i\right\}
$$

and $X=\{1, \ldots, q\}^{G}$ is endowed with the Bernoulli action $G \curvearrowright X$. Fix $F \subset \llbracket G \rrbracket$ and a basis $\left\{p_{B_{\psi_{1}}}, p_{B_{\psi_{2}}} \ldots p_{B_{\psi_{\ell}}}\right\}$ for $\operatorname{span}\left(P_{F_{ \pm}^{n}}\right)$ in $L^{\infty}(X, \mu)$ associated with $\psi_{i}: F_{\psi_{i}} \rightarrow$ $\{1, \ldots, q\}, 1 \leq i \leq \ell$.

We set

$$
\kappa=\max _{\psi, \psi_{1}, \ldots, \psi_{\ell}, a_{1}, \ldots a_{\ell} \mid p_{B_{\psi}}=\sum_{i=1}^{\ell} a_{i} p_{B_{\psi_{i}}}}\left|a_{i}\right| \geq 1 .
$$

Both $\kappa$ and $\ell$ depend on $F$ and $n$ only.

Lemma 21. Let $\sigma \in \mathrm{SA}\left(F_{n}, 4 n\left|F_{ \pm}^{n}\right|+1, \delta, d\right)$ and take a partition $\left\{A_{1}, \ldots, A_{q}\right\}$ satisfying the conclusion of Lemma 20.

If for some $\psi: F_{\psi} \rightarrow\{1, \ldots, q\}$

$$
p_{B_{\psi}}=\sum_{i=1}^{\ell} a_{i} p_{B_{\psi_{i}}}
$$

then

$$
\left\|p_{A_{\psi}}-\sum_{i=1}^{\ell} a_{i} p_{A_{\psi_{i}}}\right\|_{2}<c_{3}(F, n) \sqrt{\delta}
$$

where $c_{3}(F, n)=\left(\left(1+(3+\kappa \ell) \operatorname{Be}(\ell) N_{\ell}\right)\right) c_{2}(F, n)$ and $N_{\ell}=\ell 2^{\ell}$.

Proof. For $I \subset\{1, \ldots \ell\}$ define

$$
D_{I}:=\left\{x \in X \mid x \in B_{\psi_{i}} \Longleftrightarrow i \in I\right\}
$$

and similarly

$$
D_{I}^{\sigma}:=\left\{1 \leq k \leq d \mid k \in A_{\psi_{i}} \Longleftrightarrow i \in I\right\} .
$$

We want to bound $\left|\mu\left(D_{I}\right)-\right| D_{I}^{\sigma}|/ d|$.

Note that for any $I \subset\{1, \ldots \ell\}$

$$
\mu\left\{x \in X \mid \forall i \in I, x \in B_{\psi_{i}}\right\}=\mu\left(\bigcap_{i \in I} B_{\psi_{i}}\right) .
$$

Now either for some $s \in F_{\psi_{i_{1}}} \cap F_{\psi_{i_{2}}}$ we have $\psi_{i_{1}}(s) \neq \psi_{i_{2}}(s)$ and so $\bigcap_{i \in I} B_{\psi_{i}}=\varnothing$ or otherwise $\bigcap_{i \in I} B_{\psi_{i}}=B_{\psi^{\prime}}$ for some $\psi^{\prime}: F_{\psi^{\prime}} \rightarrow\{1, \ldots, q\}$. In the first case $\bigcap_{i \in I} A_{\psi_{i}}=$ $\varnothing$ as well and in the second case by Lemma 20

$$
\left|\mu\left(B_{\psi^{\prime}}\right)-\right| A_{\psi^{\prime}}|/ d|<c_{2}(F, n) \delta
$$


and

$$
\left|A_{\psi^{\prime}}\right| / d=\left|\bigcap_{i \in I} A_{\psi_{i}}\right| / d=\left|\left\{1 \leq k \leq d \mid \forall i \in I, k \in A_{\psi_{i}}\right\}\right| / d
$$

So in general we conclude that $\mu\left\{x \in X \mid \forall i \in I, x \in B_{\psi_{i}}\right\}$ is within $c_{1}(F, n) \delta$ of $\left|\left\{1 \leq k \leq d \mid \forall i \in I, k \in A_{\psi_{i}}\right\}\right| / d$. By inclusion-exclusion

$$
\mu\left(D_{I}\right)=\sum_{k=|I|}^{\ell} \sum_{\substack{I^{\prime} \supset I \\\left|I^{\prime}\right|=k}}(-1)^{k-|I|} \mu\left(\left\{x \in X \mid \forall i \in I^{\prime}, x \in B_{\psi_{i}}\right\}\right)
$$

and similarly

$$
\frac{\left|D_{I}^{\sigma}\right|}{d}=\sum_{k=|I|}^{\ell} \sum_{\substack{I^{\prime} \supset I \\\left|I^{\prime}\right|=k}}(-1)^{k-|I|} \frac{1}{d}\left|\left\{1 \leq k \leq d \mid \forall i \in I^{\prime}, k \in A_{\psi_{i}}\right\}\right|
$$

so by applying the triangle inequality at most $N_{\ell}=\ell 2^{\ell}$ times we have

$$
\left|\mu\left(D_{I}\right)-\right| D_{I}^{\sigma}|/ d| \leq c_{2}(F, n) N_{\ell} \delta
$$

Now

$$
\mu\left(B_{\psi}\right)=\sum_{I \subset\{1, \ldots \ell\} ; \sum_{i \in I} a_{i}=1} \mu\left(D_{I}\right)
$$

So if $\sum_{i \in I} a_{i}=1$ then $\mu\left(\bigcap_{i \in I} B_{\psi_{i}}\right)=\mu\left(\bigcap_{i \in I} B_{\psi_{i}} \cap B_{\psi}\right)$ so by a similar argument $\mu\left\{x \in B_{\psi} \mid \forall i \in I, x \in B_{\psi_{i}}\right\}$ is within $c_{2}(F, n) \delta$ of $\left|\left\{k \in A_{\psi} \mid \forall i \in I, k \in A_{\psi_{i}}\right\}\right| / d$ So if we let

$$
\tilde{D}_{I}^{\sigma}:=\left\{k \in A_{\psi} \mid x \in A_{\psi_{i}} \Longleftrightarrow i \in I\right\}
$$

then

$$
\left|\mu\left(D_{I}\right)-\right| \tilde{D}_{I}^{\sigma}|/ d| \leq c_{2}(F, n) N_{\ell} \delta
$$

so

$$
\left|\frac{\left|D_{I}^{\sigma}\right|}{d}-\frac{\left|\tilde{D}_{I}^{\sigma}\right|}{d}\right| \leq 2 c_{2}(F, n) N_{\ell} \delta
$$


Let $a(I):=\sum_{i \in I} a_{i}$.

$$
\begin{aligned}
& \left\|p_{A_{\psi}}-\sum_{i=1}^{\ell} a_{i} p_{A_{\psi_{i}}}\right\|_{2} \\
& =\left\|p_{A_{\psi}}-\sum_{I \subset\{1, \ldots \ell\}}\left(\sum_{i \in I} a_{i}\right) p_{D_{I}^{\sigma}}\right\|_{2} \\
& \leq\left\|p_{A_{\psi}}-\sum_{a(I)=1} p_{D_{I}^{\sigma}}\right\|_{2}+\left\|\sum_{a(I) \notin\{0,1\}}\left(\sum_{i \in I} a_{i}\right) p_{D_{I}^{\sigma}}\right\|_{2} \\
& \leq\left\|p_{A_{\psi}}-\sum_{a(I)=1} p_{\tilde{D}_{I}^{\sigma}}\right\|_{2}+\left\|\sum_{a(I)=1} p_{\tilde{D}_{I}^{\sigma}}-\sum_{a(I)=1} p_{D_{I}^{\sigma}}\right\|_{2}+\kappa \ell \sum_{a(I) \notin\{0,1\}}\left|D_{I}^{\sigma}\right| / d \\
& \leq \sqrt{\left|\frac{\left|A_{\psi}\right|}{d}-\sum_{a(I)=1} \frac{\left|\tilde{D}_{I}^{\sigma}\right|}{d}\right|}+\sum_{a(I)=1} \sqrt{\left|\frac{\left|\tilde{D}_{I}^{\sigma}\right|}{d}-\frac{\left|D_{I}^{\sigma}\right|}{d}\right|}+\kappa \ell \operatorname{Be}(\ell) c_{2}(F, n) \delta N_{\ell} \\
& \leq \sqrt{\left|\frac{\left|A_{\psi}\right|}{d}-\mu\left(B_{\psi}\right)\right|+\left|\sum_{a(I)=1} \mu\left(D_{I}\right)-\sum_{a(I)=1} \frac{\left|\tilde{D}_{I}^{\sigma}\right|}{d}\right|} \\
& +\sqrt{\operatorname{Be}(\ell) 2 c_{2}(F, n) \delta N_{\ell}}+\kappa \ell \operatorname{Be}(\ell) c_{2}(F, n) \delta N_{\ell} \\
& \leq \sqrt{c_{2}(F, n) \delta+\sum_{a(I)=1}\left|\mu\left(D_{I}\right)-\frac{\left|\tilde{D}_{I}^{\sigma}\right|}{d}\right|}+\sqrt{\mathrm{Be}(\ell) 2 c_{2}(F, n) \delta N_{\ell}}+\kappa \ell \mathrm{Be}(\ell) c_{2}(F, n) \delta N_{\ell} \\
& \leq c_{2}(F, n) \sqrt{\delta}+(3+\kappa \ell) B e(\ell) N_{\ell} c_{2}(F, n) \sqrt{\delta} \\
& =c_{3}(F, n) \sqrt{\delta} \text {. }
\end{aligned}
$$

\section{SofiC Dimension AND GROUPOID ACTIONS}

We now briefly recall the group action formulation of $s(G \ltimes X)$ given in DKP2, Section 5] rephrasing it here in the framework of groupoid actions.

Let $G \curvearrowright X$ be a pmp action of a pmp groupoid. Let $1 \in F \subset \llbracket G \rrbracket$ and $P$ be a partition of $X$. We write $\operatorname{HA}(F, P, n, \delta, d)$ for the set of all pairs $(\sigma, \varphi)$ where $\sigma \in \mathrm{SA}(F, n, \delta, d)$ and $\varphi$ is a map

$$
\varphi: \Sigma P_{F_{ \pm}^{n}} \rightarrow \llbracket d \rrbracket
$$

defined on $\boldsymbol{\Sigma} P_{F_{ \pm}^{n}} \subset \llbracket G \rrbracket$ and satisfying

(i) $|\operatorname{tr} \circ \varphi(p)-\mu(p)|<\delta$ for all $p \in P_{F_{ \pm}^{n}}$

(ii) $|\varphi \circ s(p)-\sigma(s) \circ \varphi(p)|<\delta$ for all $p \in P$ and $s \in F_{ \pm}^{n}$

(iii) $\left|\varphi\left(p_{1} p_{2}\right)-\varphi\left(p_{1}\right) \varphi\left(p_{2}\right)\right|<\delta$ for all $p_{1}, p_{2}, p_{1} p_{2} \in \boldsymbol{\Sigma} P_{F_{ \pm}^{n}}$.

(iv) $\left|\varphi\left(p_{X}\right)-p_{d}\right|<\delta$, where $p_{d}:=p_{\{1, \ldots, d\}}$.

(Note that since $P$ partitions $X$ and $F$ contains the identity we have $p_{X} \in \operatorname{span} P_{F_{ \pm}^{n}}$.)

We observe that if $\varphi$ satisfies these conditions then it is automatically approximately linear: 
Lemma 22. If $(\sigma, \varphi) \in \operatorname{HA}(F, 4 n, \delta, d)$ then

$$
\left|\varphi\left(p_{1}+p_{2}\right)-\left(\varphi\left(p_{1}\right)+\varphi\left(p_{2}\right)\right)\right|<146 \delta
$$

for all $p_{1}, p_{2}, p_{1}+p_{2} \in \boldsymbol{\Sigma} P_{F_{ \pm}^{n}}$ with $p_{1} p_{2}=0$ and $\varphi\left(p_{1}\right)+\varphi\left(p_{2}\right)$ is defined as in DKP1, Def. 3.3].

Proof. Using $\left(p_{1}+p_{2}\right) p_{i}=p_{i}$ we have

$$
\left|\varphi\left(p_{i}\right)-\varphi\left(p_{1}+p_{2}\right) \varphi\left(p_{i}\right)\right|<\delta
$$

for $i=1,2$. Let $\pi_{i}\left(\varphi\left(p_{1}\right), \varphi\left(p_{2}\right)\right)$ be defined as in [DKP1, Def. 3.3] so

$$
\varphi\left(p_{1}\right)+\varphi\left(p_{2}\right):=\varphi\left(p_{1}\right) \pi_{1}\left(\varphi\left(p_{1}\right), \varphi\left(p_{2}\right)\right)+\varphi\left(p_{2}\right) \pi_{2}\left(\varphi\left(p_{1}\right), \varphi\left(p_{2}\right)\right) .
$$

By [DKP1, Lemma 3.4] using the approximate homomorphism property of $\varphi$ we obtain

$$
\left|\pi_{i}\left(p_{1}, p_{2}\right)-\varphi\left(p_{i}\right) \varphi\left(p_{i}\right)^{-1}\right|<40 \delta
$$

for $i=1,2$. Since $\left|\varphi\left(p_{i}\right)-\varphi\left(p_{i}\right) \varphi\left(p_{i}\right)^{-1}\right|<8 \delta$ we obtain

$$
\left|\varphi\left(p_{1}+p_{2}\right) \pi_{i}\left(p_{1}, p_{2}\right)-\varphi\left(p_{i}\right)\right|<48 \delta
$$

and so

$$
\left|\varphi\left(p_{1}+p_{2}\right) \pi\left(p_{1}, p_{2}\right)-\left(\varphi\left(p_{1}\right)+\varphi\left(p_{2}\right)\right)\right|<48 \delta
$$

with $\pi\left(p_{1}, p_{2}\right):=\pi_{1}\left(\varphi\left(p_{1}\right), \varphi\left(p_{2}\right)\right)+\pi_{2}\left(\varphi\left(p_{1}\right), \varphi\left(p_{2}\right)\right)$.

Then

$$
\begin{aligned}
\operatorname{tr}\left(\varphi\left(p_{1}+p_{2}\right) \pi\left(p_{1}, p_{2}\right)\right) & \left.=\operatorname{tr}\left(\varphi\left(p_{1}+p_{2}\right) \pi_{1}\left(p_{1}, p_{2}\right)\right)\right)+\operatorname{tr}\left(\varphi\left(p_{1}+p_{2}\right) \pi_{2}\left(p_{1}, p_{2}\right)\right) \\
& >\operatorname{tr}\left(\varphi\left(p_{1}\right)\right)+\operatorname{tr}\left(\varphi\left(p_{2}\right)\right)-96 \delta \\
& >\tau\left(p_{1}\right)+\tau\left(p_{2}\right)-98 \delta \\
& =\tau\left(p_{1}+p_{2}\right)-98 \delta
\end{aligned}
$$

Therefore

$$
\left|\varphi\left(p_{1}+p_{2}\right)-\left(\varphi\left(p_{1}\right)+\varphi\left(p_{2}\right)\right)\right|<146 \delta .
$$

(Similarly one can also show that $\varphi(p)$ is approximately a projection for every $p \in \Sigma P_{F_{ \pm}^{n}}$.)

Definition 23. Given $E, Q, F, P, n$ and $\delta$ define successively

$$
\begin{aligned}
s_{E, Q}(F, P, n, \delta) & :=\limsup _{d \rightarrow \infty} \frac{1}{d \log (d)} \log \left(|\operatorname{HA}(F, P, n, \delta, d)|_{E, Q}\right) \\
s_{E, Q}(F, P, n) & :=\inf _{\delta>0} s_{E, Q}(F, P, n, \delta) \\
s_{E, Q}(F, P) & :=\inf _{n \in \mathbb{N}} s_{E, Q}(F, P, n)
\end{aligned}
$$

If $K \subset \llbracket G \rrbracket$ is a transversally generating set and $R$ is a dynamically generating family of projections of $L^{\infty}(X, \mu)$ define

$$
s(K, R):=\sup _{E} \sup _{Q} \inf _{F} \inf _{P} s_{E, Q}(F, P)
$$

where $E$ and $F$ range over finite subsets of $K$ and $P, Q$ range over finite subpartitions of $R$. Set $s(G, X)=s\left(\llbracket G \rrbracket, L^{\infty}(X,\{0,1\})\right)$.

Since $\llbracket G \rrbracket \cup L^{\infty}(X,\{0,1\})$ is a transversally generating set of the crossed product groupoid we obtain by Theorem 9 (if the action $G \curvearrowright X$ is essentially free) and [DKP2, Theorem 2.11] in general (compare [DKP2, Proposition 5.2]):

Proposition 24. $s(G \ltimes X)=s(G, X)$. 
Moreover if $F$ is a finite transversally generating subset of $\llbracket G \rrbracket$ and $P$ is a finite and dynamically generating partition of unity, then $s(G \ltimes X)=s_{F, P}(F, P)$.

Proposition 25. $s(G \ltimes X) \leq s(G)$.

Proof. For every $(\sigma, \varphi) \in \operatorname{HA}(F, P, n, \delta, d)$ there are at most $|Q|^{d}$ restrictions $\left.\varphi\right|_{Q}$ and hence

$$
|\mathrm{HA}(F, P, n, \delta, d)|_{E, Q} \leq|Q|^{d}|\mathrm{SA}(F, n, \delta, d)|_{E}
$$

so we have $s_{E, Q}(F, P) \leq s_{E}(F)$ and the result follows directly by the proposition above.

Remark 26. The set HA differs from the set HA introduced in [DKP2] in that we do not assume $\varphi$ to be a strict homomorphism. Furthermore the maps in DKP2] are defined on $L^{\infty}(X)$ with values in $\mathrm{M}_{d}$ using the 2-norm. It is often convenient to adopt the latter point of view for computational purposes and we will do so below. The definition of HA given above has the advantage of being purely finitary, in the spirit of the sofic property. We have maintained the notation HA in view of (iii) and (iv).

\section{The computation of $s\left(G \ltimes\left(X_{0}, \mu_{0}\right)^{G}\right)$}

Lemma 27. If $G \curvearrowright\{1, \ldots, q\}^{G}$, then $s\left(G \ltimes\left(\{1, \ldots, q\}, \mu_{0}\right)^{G}\right)=s(G)$ for any probability measure $\mu_{0}$ on $\{1, \ldots, q\}$ and pmp groupoid $G$. The same holds true for $\underline{s}$ and $s^{\omega}$.

Proof. Let $X=\{1, \ldots, q\}^{G}$. By Proposition 25] we have to prove $s(G \ltimes X) \geq s(G)$. Let $P=\left\{p_{B_{i}}\right\}$ where $B_{i}=\left\{x \in\{1, \ldots, q\}^{G} \mid x_{e}=i\right\}$. Let $\left\{p_{B_{\psi_{1}}}, p_{B_{\psi_{2}}} \ldots p_{B_{\psi_{\ell}}}\right\}$ be a basis for $\operatorname{span}\left(P_{F_{ \pm}^{n}}\right) \subset L^{\infty}(X, \mu)$, where $\psi_{i}: F_{\psi_{j}} \rightarrow\{1, \ldots, q\}$. Let $\sigma \in$ $\mathrm{SA}\left(F_{n}, 4 n\left|F_{ \pm}^{n}\right|+1, \delta, d\right)$. Let $\kappa$ be as defined before Lemma 21] Let

$$
\begin{gathered}
\gamma_{1}=\min \left\{\left|\sum_{i \in T} a_{i}\right|: \psi, \psi_{1}, \ldots . \psi_{\ell}, a_{1} \ldots, a_{\ell}, T \subset\{1, \ldots, \ell\} \text { and } p_{B_{\psi}}=\sum_{i=1}^{\ell} a_{i} p_{B_{\psi_{i}}}\right\} /\{0\} \\
\gamma_{2}=\min \left\{\left|\sum_{i \in T} a_{i}-1\right|: \psi, \psi_{1}, \ldots . \psi_{\ell}, a_{1} \ldots, a_{\ell}, T \subset\{1, \ldots, \ell\} \text { and } p_{B_{\psi}}=\sum_{i=1}^{\ell} a_{i} p_{B_{\psi_{i}}}\right\} /\{0\} \\
\gamma_{2}=\min \left\{\left|\sum_{i \in T, j \in T^{\prime}} a_{i} b_{j}\right|: \psi, \psi^{\prime} \psi_{1}, \ldots . \psi_{\ell}, a_{1} \ldots, a_{\ell}, T, T^{\prime} \subset\{1, \ldots, \ell\}\right. \text { and } \\
\left.p_{B_{\psi}}=\sum_{i=1}^{\ell} a_{i} p_{B_{\psi_{i}}}, p_{B_{\psi^{\prime}}}=\sum_{i=1}^{\ell} b_{i} p_{B_{\psi_{i}}}\right\} /\{0\} \\
\gamma=\min \left\{\gamma_{1}, \gamma_{2}, \gamma_{3}\right\}
\end{gathered}
$$

so that $\gamma$ depends only on $F$ and $n$ and $\gamma \leq 1$. We want to find $\varphi$ such that

$$
(\sigma, \varphi) \in \operatorname{HA}\left(F, P, n, 9\left|P_{F_{ \pm}^{n}}\right|^{2} \frac{1}{\gamma^{2}} \kappa^{5} \ell^{2} q c_{3}(F, n)^{2} \sqrt{\delta}, d\right)
$$

(for sufficiently large $d$ ). Using Proposition 24 this will complete the proof.

Take a partition $\left\{A_{1}, \ldots, A_{q}\right\}$ such that the conclusion of Lemma 20 holds (namely a random partition for $d$ large). For each $p_{B_{\psi_{i}}}, i=1 \ldots \ell$, let

$$
\varphi_{0}\left(p_{B_{\psi_{i}}}\right):=p_{A_{\psi_{i}}}
$$


and extend $\varphi_{0}$ linearly to $\operatorname{span}\left(P_{F_{ \pm}^{n}}\right) \subset L^{\infty}(X, \mu)$ with values in $\mathrm{M}_{d}$. We will check that $\varphi_{0}$ satisfies the following properties, where $\delta_{0}=3 \frac{1}{\gamma} \kappa^{2} \ell^{2} q c_{3}(F, n)^{2} \sqrt{\delta}$ (compare Remark 26):

(1) $\left|\operatorname{tr} \circ \varphi_{0}(p)-\mu(p)\right|<\delta_{0}$ for all $p \in P_{F_{ \pm}^{n}}$

(2) $\left\|\varphi_{0} \circ s(p)-\sigma(s) \circ \varphi_{0}(p)\right\|_{2}<\delta_{0}$ for all $p \in P$ and $s \in F_{ \pm}^{n}$

(3) $\left\|\varphi_{0}\left(p_{1} p_{2}\right)-\varphi_{0}\left(p_{1}\right) \varphi_{0}\left(p_{2}\right)\right\|_{2}<\delta_{0}$ for all $p_{1}, p_{2} \in \operatorname{span} P_{F_{ \pm}^{n}}$

(4) $\left\|\varphi_{0}\left(p_{X}\right)-p_{d}\right\|_{2}<\delta_{0}$, where $p_{d}:=p_{\{1, \ldots, d\}}$.

One can see (as shown below) that properties (1)-(4) are closely related to the properties (i)-(iv) defined above.

(1) Note for any $x \in\{1 \ldots, d\}, \psi$ and $a_{1}, \ldots a_{n}$ if

$$
\left|\left(\sum_{i=1}^{\ell} a_{i} p_{A_{\psi_{i}}}-p_{A_{\psi}}\right) x\right| \neq 0
$$

then

$$
\left|\left(\sum_{i=1}^{\ell} a_{i} p_{A_{\psi_{i}}}-p_{A_{\psi}}\right) x\right| \geq \min \left\{\gamma_{1}, \gamma_{2}\right\} \geq \gamma .
$$

Let $p_{B_{\psi}} \in P_{F_{ \pm}^{n}}$ say $p_{B_{\psi}}=\sum_{i=1}^{\ell} a_{i} p_{B_{\psi_{i}}}$ then

$$
\varphi_{0}\left(p_{B_{\psi}}\right)=\sum_{i=1}^{\ell} a_{i} p_{A_{\psi_{i}}}
$$

so

$$
\begin{aligned}
\left|\operatorname{tr} \circ \varphi_{0}\left(p_{B_{\psi}}\right)-\mu\left(p_{B_{\psi}}\right)\right| & \leq\left|\operatorname{tr}\left(\sum_{i=1}^{\ell} a_{i} p_{A_{\psi_{i}}}\right)-\operatorname{tr} \circ p_{A_{\psi}}\right|+\left|\frac{\left|A_{\psi}\right|}{d}-\mu\left(B_{\psi}\right)\right| \\
& \leq \frac{1}{\gamma}\left\|\sum_{i=1}^{\ell} a_{i} p_{A_{\psi_{i}}}-p_{A_{\psi}}\right\|_{2}^{2}+c_{2}(F, n) \delta \\
& \leq \frac{1}{\gamma} c_{3}(F, n)^{2} \delta^{2}+c_{2}(F, n) \delta \leq \frac{1}{\gamma} c_{3}(F, n)^{2} \sqrt{\delta}, \text { for } \delta<1
\end{aligned}
$$

where we use both Lemma 20 and 21

(2) Here we use Lemma 21 Let $s \in F_{ \pm}^{n}$ and suppose

$$
s p_{B_{i}}=\sum_{i=1}^{\ell} a_{i} p_{B_{\psi_{i}}} .
$$

Then

$$
\varphi_{0}\left(s p_{B_{i}}\right)=\sum_{i=1}^{\ell} a_{i} p_{A_{\psi_{i}}}
$$

and

$$
\left\|\sum_{i=1}^{\ell} a_{i} p_{A_{\psi_{i}}}-\sigma(s) p_{A_{i}}\right\|_{2}<c_{3}(F, n) \sqrt{\delta} .
$$

On the other hand

$$
p_{B_{i}}=\sum_{i=1}^{\ell} b_{i} p_{B_{\psi_{i}}} \Rightarrow \varphi_{0}\left(p_{B_{i}}\right)=\sum_{i=1}^{\ell} b_{i} p_{A_{\psi_{i}}}
$$

and

$$
\left\|\sum_{i=1}^{\ell} b_{i} p_{A_{\psi_{i}}}-\sigma(1) p_{A_{i}}\right\|_{2}<c_{3}(F, n) \sqrt{\delta} .
$$


Thus

$$
\begin{aligned}
& \left\|\varphi_{0}\left(s p_{B_{i}}\right)-\sigma(s) \varphi_{0}\left(p_{B_{i}}\right)\right\|_{2} \\
& \leq\left\|\varphi_{0}\left(s p_{B_{i}}\right)-\sigma(s) p_{A_{i}}\right\|_{2}+\left\|\sigma(s) p_{A_{i}}-\sigma(s) \sigma(1) p_{A_{i}}\right\|_{2} \\
& +\left\|\sigma(s) \sigma(1) p_{A_{i}}-\sigma(s) \varphi_{0}\left(p_{B_{i}}\right)\right\|_{2} \\
& <2 c_{3}(F, n) \sqrt{\delta}+\sqrt{\delta}=3 c_{3}(F, n) \sqrt{\delta}
\end{aligned}
$$

(3) Let $p_{1}=\sum_{i=1}^{\ell} a_{i} p_{B_{\psi_{i}}}$ and $p_{2}=\sum_{i=1}^{\ell} b_{i} p_{B_{\psi_{i}}}$ then

$$
\begin{aligned}
\left\|\varphi_{0}\left(p_{1} p_{2}\right)-\varphi_{0}\left(p_{1}\right) \varphi_{0}\left(p_{2}\right)\right\|_{2} & =\left\|\sum_{i, j=1}^{\ell} a_{i} b_{i}\left(\varphi_{0}\left(p_{B_{\psi_{i}}} p_{B_{\psi_{j}}}\right)-p_{A_{\psi_{i}}} p_{A_{\psi_{j}}}\right)\right\|_{2} \\
& \leq \kappa^{2} \sum_{i, j=1}^{\ell}\left\|\varphi_{0}\left(p_{B_{\psi_{i}}} p_{B_{\psi_{j}}}\right)-p_{A_{\psi_{i}}} p_{A_{\psi_{j}}}\right\|_{2}
\end{aligned}
$$

So it is enough to show that for all $i, j$

$$
\left\|\varphi_{0}\left(p_{B_{\psi_{i}}} p_{B_{\psi_{j}}}\right)-p_{A_{\psi_{i}}} p_{A_{\psi_{j}}}\right\|_{2}<c_{3}(F, n) \sqrt{\delta} \text {. }
$$

But as we have seen in the proof of Lemma 21] either $p_{B_{\psi_{i}}} p_{B_{\psi_{j}}}$ and $p_{A_{\psi_{i}}} p_{A_{\psi_{j}}}$ are both 0 in which case we are done or $p_{B_{\psi_{i}}} p_{B_{\psi_{j}}}=p_{B \psi^{\prime}}$ and $p_{A_{\psi_{i}}} p_{A_{\psi_{j}}}=p_{A_{\psi^{\prime}}}$ for some $\psi^{\prime}$ in which case if $p_{B_{\psi^{\prime}}}=\sum_{i=1}^{\ell} c_{i} p_{B_{\psi_{i}}}$ then by Lemma 21

$$
\left\|\varphi_{0}\left(p_{B_{\psi_{i}}} p_{B_{\psi_{j}}}\right)-p_{A_{\psi_{i}}} p_{A_{\psi_{j}}}\right\|_{2}=\left\|\sum_{i=1}^{\ell} c_{i} p_{A_{\psi_{i}}}-p_{A_{\psi^{\prime}}}\right\|_{2}<c_{3}(F, n) \sqrt{\delta}
$$

(4) $p_{X}=\sum_{i=1}^{q} p_{B_{i}}$ and $I_{d}=\sum_{i=1}^{q} p_{A_{i}}$ so by the triangle inequality it suffices to show

By Lemma 21

$$
\left\|\varphi_{0}\left(p_{B_{i}}\right)-p_{A_{i}}\right\|_{2}<2 c_{3}(F, n) \sqrt{\delta}
$$

$$
\left\|\varphi_{0}\left(p_{B_{i}}\right)-\sigma(1) p_{A_{i}}\right\|_{2}<c_{3}(F, n) \sqrt{\delta}
$$

so

$$
\begin{aligned}
\left\|\varphi_{0}\left(p_{B_{i}}\right)-p_{A_{i}}\right\|_{2} \leq & \left\|\varphi_{0}\left(p_{B_{i}}\right)-\sigma(1) p_{A_{i}}\right\|_{2}+\left\|\sigma(1) p_{A_{i}}-p_{A_{i}}\right\|_{2} \\
& <2 c_{3}(F, n) \sqrt{\delta}+\sqrt{\delta}<2 c_{3}(F, n) \sqrt{\delta}
\end{aligned}
$$

We now show how to define $\varphi$ using $\varphi_{0}$. For each $p_{B_{\psi}}$

$$
\left\|\varphi_{0}\left(p_{B_{\psi}}\right)-p_{A_{\psi}}\right\|_{2} \leq c_{3}(F, n) \sqrt{\delta}
$$

by Lemma 21. Let $M$ be the number of $x \in\{1 \ldots, d\}$ such that $\varphi_{0}\left(p_{B_{\psi}}\right) x=p_{A_{\psi}} x$ then

hence

$$
\| \varphi_{0}\left(p_{B_{\psi}}\right)-p_{A_{\psi} \|_{2}} \geq \sqrt{\frac{d-M}{d} \gamma^{2}}
$$

$$
M \geq d\left(1-c_{3}(F, n)^{2} \frac{1}{\gamma^{2}} \delta\right)
$$

Similarly for $p_{B_{\psi}}, p_{B_{\psi^{\prime}}}$ we have

$$
\left\|\varphi_{0}\left(p_{B_{\psi}}\right) \varphi_{0}\left(p_{B_{\psi^{\prime}}}\right)-\varphi\left(p_{B_{\psi}}\right) \varphi\left(p_{B_{\psi^{\prime}}}\right)\right\|_{2} \leq 2 \kappa^{2} c_{3}(F, n) \sqrt{\delta}
$$


by (3) so

$$
\left|\left\{x \in[d]: \varphi_{0}\left(p_{B_{\psi}}\right) \varphi_{0}\left(p_{B_{\psi^{\prime}}}\right) x=0\right\}\right| \geq d\left(1-c_{3}(F, n)^{2} \kappa^{4} \delta \frac{1}{\gamma^{2}}\right)
$$

Let $V$ be the set of all $x \in\{1, \ldots d\}$ such that for all $p_{B_{\psi}}$ (arbitrarily represented) we have

$$
\varphi_{0}\left(p_{B_{\psi}}\right) x=p_{A_{\psi}} x
$$

and for all $p_{B_{\psi}}, p_{B_{\psi^{\prime}}}$ with $p_{B_{\psi}} p_{B_{\psi^{\prime}}}=0$ we have

$$
\varphi_{0}\left(p_{B_{\psi}}\right) \varphi_{0}\left(p_{B_{\psi^{\prime}}}\right) x=0
$$

then

$|V| \geq d\left(1-\left|P_{F_{ \pm}^{n}}\right| c_{3}(F, n)^{2} \delta \frac{1}{\gamma^{2}}-\left|P_{F_{ \pm}^{n}}\right|^{2} c_{3}(F, n)^{2} \kappa^{4} \delta \frac{1}{\gamma^{2}}\right) \geq d\left(1-2\left|P_{F_{ \pm}^{n}}\right|^{2} c_{3}(F, n)^{2} \kappa^{4} \delta \frac{1}{\gamma^{2}}\right)$

and for any $\left.p \in \Sigma P_{F_{ \pm}^{n}} \varphi_{0}(p)\right|_{V} \subset \llbracket d \rrbracket$. We finally define

$$
\begin{aligned}
\varphi: \Sigma P_{F_{ \pm}^{n}} & \rightarrow \llbracket d \rrbracket \\
p & \left.\mapsto \varphi_{0}(p)\right|_{V}
\end{aligned}
$$

Let us check that $\varphi$ satisfies (i)-(iv):

(i)

(ii)

$$
\begin{aligned}
\left|\operatorname{tr} \circ \varphi\left(p_{B_{\psi}}\right)-\mu\left(B_{\psi}\right)\right| & \leq\left|\operatorname{tr} \circ \varphi\left(p_{B_{\psi}}\right)-\operatorname{tr} \circ \varphi_{0}\left(p_{B_{\psi}}\right)\right|+\delta_{0} \\
& \leq \kappa \ell 2\left|P_{F_{ \pm}^{n}}\right|^{2} c_{3}(F, n)^{2} \kappa^{4} \delta \frac{1}{\gamma^{2}}+\delta_{0}
\end{aligned}
$$

(iii)

$$
\begin{aligned}
&\left|\varphi\left(s p_{B_{i}}\right)-\sigma(1) \varphi\left(p_{B_{i}}\right)\right| \leq\left\|\varphi\left(s p_{B_{i}}\right)-\sigma(1) \varphi\left(p_{B_{i}}\right)\right\|_{2} \\
& \leq\left\|\varphi\left(s p_{B_{i}}\right)-\varphi_{0}\left(s p_{B_{i}}\right)\right\|_{2} \\
&+\left\|\sigma(1) \varphi\left(p_{B_{i}}\right)-\sigma(1) \varphi_{0}\left(p_{B_{i}}\right)\right\|_{2}+\delta_{0} \\
& \leq \kappa \ell 4\left|P_{F_{ \pm}^{n}}\right| c_{3}(F, n) \kappa^{2} \sqrt{\delta} \frac{1}{\gamma}+\delta_{0}
\end{aligned}
$$

$$
\begin{aligned}
\left|\varphi\left(p_{1}\right) \varphi\left(p_{2}\right)-\varphi\left(p_{1} p_{2}\right)\right| & \leq\left\|\varphi\left(p_{1}\right) \varphi\left(p_{2}\right)-\varphi\left(p_{1} p_{2}\right)\right\|_{2} \\
& \leq\left\|\varphi\left(p_{1}\right) \varphi\left(p_{2}\right)-\varphi_{0}\left(p_{1}\right) \varphi_{0}\left(p_{2}\right)\right\|_{2} \\
& +\left\|\varphi\left(p_{1} p_{2}\right)-\varphi_{0}\left(p_{1} p_{2}\right)\right\|_{2}+\delta_{0} \\
& \leq \kappa^{2} \ell^{2} q 4\left|P_{F_{ \pm}^{n}}\right| c_{3}(F, n) \kappa^{2} \sqrt{\delta} \frac{1}{\gamma}+\delta_{0}
\end{aligned}
$$

(iv)

$$
\left|\varphi\left(p_{X}\right)-p_{d}\right| \leq \kappa \ell 2\left|P_{F_{ \pm}^{n}}\right| c_{3}(F, n) \kappa^{2} \sqrt{\delta} \frac{1}{\gamma}+\delta_{0}
$$

Thus for every $\sigma \in \mathrm{SA}\left(F_{n}, 4 n\left|F_{ \pm}^{n}\right|+1, \delta, d\right)$ with $d$ sufficiently large we found $\varphi$ so that $(\sigma, \varphi) \in \operatorname{HA}\left(F, P, n, 9\left|P_{F_{ \pm}^{n}}\right|^{2} \frac{1}{\gamma^{2}} \kappa^{5} \ell^{2} q c_{3}(F, n)^{2} \sqrt{\delta}, d\right)$.

We now check that

$$
s(G)=\sup _{E} \inf _{F} \inf _{n \in \mathbb{N}} \inf _{\delta>0} \limsup _{d \rightarrow \infty} \frac{1}{d \log d}\left|\operatorname{SA}\left(F_{n}, 4 n\left|F_{ \pm}^{n}\right|+1, \delta, d\right)\right|_{E}
$$

Recall that $F_{n}:=F \cup\left\{p_{F_{0 \mid \pi}} \mid F_{0} \subset F_{ \pm}^{n}, \pi\right.$ partition of $\left.F_{0}\right\}$. 
The right hand side equals

$$
\sup _{E} \inf _{F} \inf _{n \in \mathbb{N}} s_{E}\left(F_{n}, 4 n\left|F_{ \pm}^{n}\right|+1\right) .
$$

Let $\epsilon>0$ and choose $n_{0}$ so that

$$
\left|s_{E}\left(F_{n_{0}}, 4 n_{0}\left|F_{ \pm}^{n_{0}}\right|+1\right)-\inf _{n} s_{E}\left(F_{n}, 4 n\left|F_{ \pm}^{n}\right|+1\right)\right|<\varepsilon
$$

and

$$
\left|S_{E}\left(F, 4 n_{0}\left|F_{ \pm}^{n_{0}}\right|+1\right)-\inf _{n} s_{E}\left(F, 4 n\left|F_{ \pm}^{n}\right|+1\right)\right|<\varepsilon
$$

Clearly $s_{E}\left(F_{n_{0}}, 4 n_{0}\left|F_{ \pm}^{n_{0}}\right|+1\right) \leq s_{E}\left(F, 4 n_{0}\left|F_{ \pm}^{n_{0}}\right|+1\right)$ but on the other hand if $\sigma \epsilon$ $\mathrm{SA}\left(F_{n_{0}}, 4 n_{0}\left|\left(F_{n_{0}}\right)_{ \pm}^{n_{0}}\right|+1, \delta, d\right)$ then $\sigma \in \mathrm{SA}\left(F_{n_{0}}, 4 n_{0}\left|F_{ \pm}^{n_{0}}\right|+1, \delta, d\right)$ since $4 n_{0}\left|\left(F_{n_{0}}\right)_{ \pm}^{n_{0}}\right|+$ $1 \geq 4 n_{0}\left|F_{ \pm}^{n_{0}}\right|+1$. So

$$
\inf _{F} s_{E}\left(F, 4 n_{0}\left|F_{ \pm}^{n_{0}}\right|+1\right)=\inf _{F} s_{E}\left(F_{n_{0}}, 4 n_{0}\left|F_{ \pm}^{n_{0}}\right|+1\right)
$$

Since $\varepsilon$ was arbitrary

$$
\inf _{F} \inf _{n} s_{E}\left(F_{n}, 4 n\left|F_{ \pm}^{n}\right|+1\right)=\inf _{F} \inf _{n} s_{E}\left(F, 4 n\left|F_{ \pm}^{n}\right|+1\right)=s_{E}(G)
$$

since

However

$$
\inf _{n} s_{E}(F, n)=\inf _{n} s_{E}\left(F, 4 n\left|F_{ \pm}^{n}\right|+1\right) .
$$

$$
\left|\mathrm{SA}\left(F_{n}, 4 n\left|F_{ \pm}^{n}\right|+1, \delta, d\right)\right|_{E} \leq\left|\mathrm{HA}\left(F, P, n, 9\left|P_{F_{ \pm}^{n}}\right|^{2} \frac{1}{\gamma^{2}} \kappa^{5} \ell^{2} q c_{3}(F, n)^{2} \sqrt{\delta}, d\right)\right|_{E}
$$

so $s(G) \leq s(G \ltimes X)$ by Proposition 24. Replacing limsup by liminf or $\lim _{d \rightarrow \omega}$ above, we get a similar inequality for $\underline{s}$ and $s^{\omega}$.

Theorem 28. Let $G$ be a pmp groupoid, $\left(X_{0}, \mu_{0}\right)$ be a standard probability space, and $G \curvearrowright X_{0}^{G}$ be the corresponding a Bernoulli action. Then $s\left(G \ltimes X_{0}^{G}\right)=s(G)$. The same holds true for $\underline{s}$ and $s^{\omega}$.

Proof. Again we only need to prove $s(G) \leq s\left(G \ltimes X_{0}^{G}\right)$. Let $U=\left\{U_{1}, U_{2}, \ldots, U_{q}\right\}$ be any finite partition of $X_{0}$ into measurable sets. Let

$$
B_{U}=\left\{B_{U_{1}}, \ldots, B_{U_{q}}\right\}
$$

where

$$
B_{U_{i}}=\left\{x \in X_{0}^{G} \mid \forall e \in G_{0}, x_{e} \in U_{i}\right\}
$$

Let $M:=\left\{p_{U_{i}}\right\}$ for all possible choices of $U$ and all $i$, so that $M$ is dynamically generating *-subalgebra of $L^{\infty}(X, \mu)$. Now if $P_{U}:=\left\{p_{U_{1}}, \ldots, p_{U_{q}}\right\}$ then

$$
s(G \ltimes X)=\sup _{E} \sup _{Q} \inf _{F} \inf _{P_{U}} s_{E, Q}\left(F, P_{U}\right)=\sup _{E} \sup _{Q} \inf _{P_{U}} \inf _{F} s_{E, Q}\left(F, P_{U}\right)
$$

Since the map $X_{0} \rightarrow\{1 \ldots, q\}$ defined by $U_{i} \ni x \mapsto i$ extends $G$-equivariantly to $X_{0}^{G} \rightarrow\{1, \ldots, q\}^{G}$ we have

$$
\inf _{F} s(F) \leq \inf _{F} s_{E, Q}\left(F, P_{U}\right) .
$$

Hence $s(G) \leq s(G \ltimes X)$. The same holds for $\underline{s}$ and $s^{\omega}$.

Corollary 29. A pmp groupoid $G$ is s-regular if and only if the crossed product groupoid $G \ltimes X_{0}^{G}$ associated with the Bernoulli action $G \curvearrowright X_{0}^{G}$ is s-regular for any base space $\left(X_{0}, \mu_{0}\right)$. 
Proof. Since

$$
\underline{s}(G)=\underline{s}\left(G \times X_{0}^{G}\right) \leq s\left(G \times X_{0}^{G}\right)=s(G)
$$

$\underline{s}(G)=s(G)$ if and only if $\underline{s}\left(G \times X_{0}^{G}\right)=s\left(G \times X_{0}^{G}\right)$.

\section{The SCALING FORMULA}

Proposition 30 (Scaling formula). Let $G$ be an ergodic pmp groupoid, $0 \neq p \epsilon$ $L^{\infty}\left(G^{0}\right)$. Then

$$
s(G)-1=\mathfrak{h}(p)(s(p G p)-1)
$$

where $p G p$ is endowed with the normalized Haar measure $\frac{\mathfrak{h}_{\mid p G p}}{\mathfrak{h}(p)}$. Furthermore the same equality holds for $\underline{s}$ and $s_{\omega}$ therefore:

$$
G \text { is s-regular } \Leftrightarrow p G p \text { is s-regular. }
$$

We will start by showing $\geq$ in the rational case, which is easier to handle:

Lemma 31. If $\mathfrak{h}(p) \in \mathbb{Q}$ then

$$
s(p G p) \leq \frac{1}{\mathfrak{h}(p)} s(G)+1-\frac{1}{\mathfrak{h}(p)} .
$$

Furthermore the same inequality holds for $\underline{s}$ and $s_{\omega}$.

Proof. Write $\mathfrak{h}(p)=\frac{N-k}{N}$ and choose $s_{1}, s_{2} \ldots s_{k} \in \llbracket G \rrbracket$ with

$$
s_{i}^{-1} s_{i} \leq p, \mathfrak{h}\left(s_{i}^{-1} s_{i}\right)=\frac{1}{N}
$$

and

$$
\sum_{i=1}^{k} s_{i} s_{i}^{-1}=1-p
$$

Let $S=\left\{s_{1}, \ldots s_{k}\right\}$ so $p G p \cup S$ is generating $G$ and let $E, F \subset \llbracket p G p \rrbracket$ be finite subsets. We may assume that $p \in E \cap F$ and $s_{i}^{-1} s_{i} \in E \cap F$ for all $i$. Let $\sigma \in \operatorname{SA}_{p G p}(F, 4 n+$ $5, \delta, d)$. In light of [DKP2, Lemma 2.13] we may also assume that $N-k \mid d$. Let $d^{\prime}=\frac{N}{N-k} d$ and partition $\left\{1, \ldots, d^{\prime}\right\}$ into sets $A_{0} \ldots A_{k}$ with $A_{0}=\{1, \ldots d\}$ and $\left|A_{i}\right|=\frac{d^{\prime}}{N}$. Since

$$
\left|\operatorname{Fix}\left(\sigma\left(s_{i}^{-1} s_{i}\right)\right)\right| \geq \frac{\mathfrak{h}\left(s_{i}^{-1} s_{i}\right)}{\mathfrak{h}(p)} d-\delta d
$$

we may choose a subset $B_{i} \subset A_{0}$ with exactly $\frac{d^{\prime}}{N}$ elements such that

$$
\left|\operatorname{Fix}\left(\sigma\left(s_{i}^{-1} s_{i}\right)\right) \triangle B_{i}\right|<\delta d^{\prime} .
$$

For each $i=1 \ldots k$ let $\gamma\left(s_{i}\right)$ be a bijection $B_{i} \rightarrow A_{i}$ (we have $\left(\frac{d^{\prime}}{N} !\right)^{k}$ choices).

As

$$
\begin{gathered}
s_{j}^{-1} s_{i}^{-1}=s_{i} s_{j}=0 i, j \geq 1 \\
s_{i}^{-1} s_{j}=0 \quad i \neq j \geq 1 \\
s s_{i}=s_{i}^{-1} s^{-1} \quad i \geq 1, s \in p G p \\
s_{i}^{-1} s_{i} \in F
\end{gathered}
$$

each element of $\cup_{n}(\llbracket p G p \rrbracket \cup S)^{n}$ can be written (not necessarily uniquely) as $s_{i} f s_{j}^{-1}$ for $i, j \geq 0$ where $s_{0}=p$ and $f \in \llbracket p G p \rrbracket$. Let $\gamma\left(s_{0}\right)=p_{A_{0}}$ We define a map

$$
\sigma_{\gamma}:(F \cup S)_{ \pm}^{2 n+5} \rightarrow \llbracket d^{\prime} \rrbracket
$$

by

$$
\sigma_{\gamma}\left(s_{i} f s_{j}^{-1}\right):=\gamma\left(s_{i}\right) \sigma^{\prime}\left(s_{i}^{-1} s_{i} f s_{j}^{-1} s_{j}\right) \gamma\left(s_{j}\right)^{-1} \text {. }
$$


where we denote $\sigma^{\prime}(f)$ the permutation $\left(\begin{array}{rr}\sigma(f) & 0 \\ 0 & 0\end{array}\right)$ acting in $A_{0} \subset\left\{1, \ldots, d^{\prime}\right\}$. Note that $\sigma_{\gamma}$ is well defined: if $s_{i_{1}} f_{1} s_{j_{1}}^{-1}=s_{i_{2}} f_{2} s_{j_{2}}^{-1}$ then since the $s_{i}$ have disjoint ranges we have $i_{1}=i_{2} j_{1}=j_{2}$ so $s_{i_{1}} f_{1} s_{j_{1}}^{-1}=s_{i_{1}} f_{2} s_{j_{1}}^{-1}$ hence $s_{i_{1}}^{-1} s_{i_{1}} f_{1} s_{j_{1}}^{-1} s_{j_{1}}=s_{i_{1}}^{-1} s_{i_{1}} f_{2} s_{j_{1}}^{-1} s_{j_{1}}$ so that

$$
\gamma\left(s_{i_{1}}\right) \sigma^{\prime}\left(s_{i_{1}}^{-1} s_{i_{1}} f_{1} s_{j_{1}}^{-1} s_{j_{1}}\right) \gamma\left(s_{j_{1}}\right)^{-1}=\gamma\left(s_{i_{1}}\right) \sigma^{\prime}\left(s_{i_{1}}^{-1} s_{i_{1}} f_{2} s_{j_{1}}^{-1} s_{j_{1}}\right) \gamma\left(s_{j_{1}}\right)^{-1} .
$$

then extend $\sigma_{\gamma}$ linearly to $\Sigma(F \cup S)_{ \pm}^{2 n+5}$.

Let $\delta^{\prime}=5 N^{2} \delta+150 N^{2}(2|F \cup S|+1)^{2(2 n+5)} \delta$. We claim that:

$$
\sigma_{\gamma} \in \mathrm{SA}_{G}\left(F \cup S, n, \delta^{\prime}, d\right) .
$$

Let us first see what this implies. Note if $\sigma^{1}(f) \neq \sigma^{2}(f)$ then $\sigma_{\gamma}^{1}(f)=p_{A_{0}} \sigma_{\gamma}^{1}(f) p_{A_{0}}=$ $\sigma_{\gamma}^{1}(f) \neq \sigma_{\gamma}^{2}(f)$. Next note if $\gamma_{1}\left(s_{i}\right) \neq \gamma_{2}\left(s_{i}\right): B_{i} \rightarrow A_{i}$ are two bijections then if we have

$$
\sigma_{\gamma_{1}}\left(s_{i}\right)=\gamma_{1}\left(s_{i}\right) \sigma^{\prime}(p)=\gamma_{2}\left(s_{i}\right) \sigma^{\prime}(p)=\sigma_{\gamma_{2}}\left(s_{i}\right)
$$

we must have $\left.\gamma_{1}\left(s_{i}\right)\right|_{f i x(\sigma(p))}=\left.\gamma_{2}\left(s_{i}\right)\right|_{f i x(\sigma(p))}$. But since $|\operatorname{Fix}(\sigma(p)) \triangle\{1, \ldots, . d\}| \leq$ $\delta d$ we have at most $|\llbracket\lceil\delta d\rceil \rrbracket| \leq(\lceil\delta d\rceil+1)^{\lceil\delta d\rceil}$ choices for $\gamma_{2}$ such that $\gamma_{2}\left(s_{i}\right) \neq \gamma_{1}\left(s_{i}\right)$ and $\sigma_{\gamma_{2}}\left(s_{i}\right)=\sigma_{\gamma_{1}}\left(s_{i}\right)$. Therefore there are at most $(\lceil\delta d\rceil+1)^{\lceil\delta d\rceil k}$ choices for $\gamma_{2}$ such that $\left.\sigma_{\gamma_{1}}\right|_{E \cup S} \neq\left.\sigma_{\gamma_{2}}\right|_{E \cup S}$ Thus each $\sigma_{\mid E} \in \mathrm{SA}_{p G p}(F, n, \delta, d)$ gives at least $\frac{\left(\frac{d^{\prime}}{N} !\right)^{k}}{([\delta d\rceil+1)^{\lceil\delta d\rceil k}}$ distinct elements $\sigma_{\mid E \cup S}^{\prime} \in \mathrm{SA}_{G}\left(F \cup S, n, \delta^{\prime}, d^{\prime}\right)$ so we obtain

$$
\left|\mathrm{SA}_{p G p}(F, n, \delta, d)\right|_{E} \leq\left|\mathrm{SA}_{G}\left(F \cup S, 4 n+5, \delta^{\prime}, d^{\prime}\right)\right|_{E \cup S} / \frac{\left(\frac{d^{\prime}}{N} !\right)^{k}}{(\lceil\delta d\rceil+1)^{\lceil\delta d\rceil k}}
$$

So let us show that $\sigma_{\gamma} \in \mathrm{SA}_{G}\left(F \cup S, n, \delta^{\prime}, d^{\prime}\right)$. We first show that $\sigma_{\gamma}$ is approximately linear. Suppose in the span we have

$$
s_{i} f s_{j}=\sum_{\ell=1}^{M} s_{i_{\ell}} f_{\ell} s_{j_{\ell}}
$$

then if we let $L(u, v)$ be the set of all $\ell$ with $i_{\ell}=u j_{\ell}=v$ then $s_{i} f s_{j}=\sum_{\ell \in L(i, j)} s_{i} f_{\ell} s_{j}^{-1}$ and $s_{u} 0 s_{v}=0=\sum_{\ell \in L(u, v)} s_{p} f_{\ell} s_{q}^{-1}$ for $(u, v) \neq(i, j)$. So we may assume $i_{\ell}=i, j_{\ell}=j$ for all $\ell$ then

$$
\begin{aligned}
\sigma_{\gamma}\left(s_{i} f s_{j}\right) & =\gamma\left(s_{i}\right) \sigma^{\prime}\left(s_{i}^{-1} s_{i} f s_{j}^{-1} s_{j}\right) \gamma\left(s_{j}\right)^{-1} \\
& =\gamma\left(s_{i}\right) \sigma^{\prime}\left(s_{i}^{-1}\left(\sum_{\ell=1}^{M} s_{i} f_{\ell} s_{j}^{-1}\right) s_{j}\right) \gamma\left(s_{j}\right)^{-1} \\
& =\gamma\left(s_{i}\right) \sigma^{\prime}\left(\sum_{\ell=1}^{M} s_{i}^{-1} s_{i} f_{\ell} s_{j}^{-1} s_{j}\right) \gamma\left(s_{j}\right)^{-1}
\end{aligned}
$$

so by [DKP1, Lemma 3.5]

$$
\begin{aligned}
\left|\sigma_{\gamma}\left(s_{i} f s_{j}\right)-\sum_{\ell=1}^{M} \sigma_{\gamma}\left(s_{i} f_{\ell} s_{j}\right)\right| & =\left|\sigma_{\gamma}\left(s_{i} f s_{j}\right)-\sum_{\ell=1}^{M} \gamma\left(s_{i}\right) \sigma^{\prime}\left(s_{i}^{-1} s_{i} f_{\ell} s_{j}^{-1} s_{j}\right) \gamma\left(s_{j}\right)^{-1}\right| \\
& <150(2|F \cup S|+1)^{2(n+5)} \delta .
\end{aligned}
$$


Thus if $f=\sum_{\ell=1}^{M} f_{\ell}$, then

$$
\begin{aligned}
\left|\sigma_{\gamma}(f)-\sum_{\ell=1}^{M} \sigma_{\gamma}\left(f_{\ell}\right)\right| & =\left|\sigma_{\gamma}\left(\sum_{i, j=1}^{N} s_{i} f s_{j}\right)-\sum_{\ell=1}^{M} \sigma_{\gamma}\left(\sum_{i, j=1}^{N} s_{i} f_{\ell} s_{j}\right)\right| \\
& \leq \sum_{i, j=1}^{N}\left|\sigma_{\gamma}\left(s_{i} f s_{j}\right)-\sum_{\ell=1}^{M} \sigma_{\gamma}\left(s_{i} f_{\ell} s_{j}\right)\right| \\
& <150 N^{2}(2|F \cup S|+1)^{2(n+5)} \delta .
\end{aligned}
$$

Thus to show that $\sigma_{\gamma}$ is $\delta^{\prime}$-multiplicative it will suffice to show that for $a, b \in$ $(F \cup S)_{ \pm}^{n}$ such that $a b \in(F \cup S)_{ \pm}^{n}$ we have

$$
\left|\sigma_{\gamma}(a b)-\sigma_{\gamma}(a) \sigma_{\gamma}(b)\right|<5 \delta .
$$

Indeed then for $\sum_{i} a_{i}, \sum_{i} b_{i} \in \boldsymbol{\Sigma}(F \cup S)_{ \pm}^{n}$ with $\left(\sum_{i} a_{i}\right)\left(\sum_{j} b_{j}\right) \in \boldsymbol{\Sigma}(F \cup S)_{ \pm}^{n}$

$$
\begin{aligned}
& \left|\sigma_{\gamma}\left(\left(\sum_{i} a_{i}\right)\left(\sum_{j} b_{j}\right)\right)-\sigma_{\gamma}\left(\sum_{i} a_{i}\right) \sigma_{\gamma}\left(\sum_{j} b_{j}\right)\right| \\
& \leq\left|\sigma_{\gamma}\left(\sum_{i, j} a_{i} b_{j}\right)-\left(\sum_{i} \sigma_{\gamma}\left(a_{i}\right)\right)\left(\sum_{j} \sigma_{\gamma}\left(b_{j}\right)\right)\right|+\left(150 N^{2}(2|F \cup S|+1)^{2(2 n+5)} \delta\right)^{2} \\
& \quad \leq\left|\sum_{i, j} \sigma_{\gamma}\left(a_{i} b_{j}\right)-\sum_{i, j} \sigma_{\gamma}\left(a_{i}\right) \sigma_{\gamma}\left(b_{j}\right)\right|+2\left(150 N^{2}(2|F \cup S|+1)^{2(2 n+5)}\right)^{2} \delta \\
& \quad \leq 5 N^{2} \delta+2\left(150 N^{2}(2|F \cup S|+1)^{2(2 n+5)}\right)^{2} \delta \\
& \quad=\delta^{\prime}
\end{aligned}
$$

So say we have $a=s_{i_{1}} f_{1} s_{j_{1}}^{-1}$ and $b=s_{i_{2}} f_{2} s_{j_{2}}^{-1}$ where $f_{1}, f_{2} \in F_{ \pm}^{n}$. If $j_{1} \neq i_{2}$ then $s_{j_{1}}^{-1} s_{i_{2}}=\gamma\left(s_{j_{1}}\right)^{-1} \gamma\left(s_{i_{2}}\right)=0$ so we are done. Suppose otherwise, then

$$
\begin{aligned}
\mid \sigma_{\gamma}(a b)- & \sigma_{\gamma}(a) \sigma_{\gamma}(b)|=| \gamma\left(s_{i_{1}}\right) \sigma^{\prime}\left(s_{i_{1}}^{-1} s_{i_{1}} f_{1}\left(s_{j_{1}}^{-1} s_{j_{1}}\right)^{3} f_{2} s_{j_{2}}^{-1} s_{j_{2}}\right) \gamma\left(s_{j_{2}}\right)^{-1}- \\
& \gamma\left(s_{i_{1}}\right) \sigma^{\prime}\left(s_{i_{1}}^{-1} s_{i_{1}} f_{1} s_{j_{1}}^{-1} s_{j_{1}}\right) \gamma\left(s_{j_{1}}\right)^{-1} \gamma\left(s_{j_{1}}\right) \sigma^{\prime}\left(s_{j_{1}}^{-1} s_{j_{1}} f_{2} s_{j_{2}}^{-1} s_{j_{2}}\right) \gamma\left(s_{j_{2}}\right)^{-1} \mid
\end{aligned}
$$

Note first that for any $i>0$

$$
\begin{aligned}
\left|\gamma\left(s_{i}\right)^{-1} \gamma\left(s_{i}\right)-\sigma^{\prime}\left(s_{i}^{-1} s_{i}\right)\right| & \leq \frac{1}{d^{\prime}}\left|\operatorname{Fix}\left(\sigma\left(s_{i}^{-1} s_{i}\right)\right) \triangle B_{i}\right|+\left|\sigma\left(s_{i}^{-1} s_{i}\right)-p_{\operatorname{Fix}\left(\sigma\left(s_{i}^{-1} s_{i}\right)\right)}\right| \\
& \leq 2 \delta
\end{aligned}
$$

and for $g_{1}, g_{2} \in F_{ \pm}^{2 n+5}$ such that $g_{1} g_{2} \in F_{ \pm}^{2 n+5}$

$$
\left|\sigma^{\prime}\left(g_{1} g_{2}\right)-\sigma^{\prime}\left(g_{1}\right) \sigma^{\prime}\left(g_{2}\right)\right|=\frac{1}{d^{\prime}} d\left|\sigma\left(g_{1} g_{2}\right)-\sigma\left(g_{1}\right) \sigma\left(g_{2}\right)\right| \leq \delta
$$

so by repeated applications of the triangle inequality

$$
\left|\sigma_{\gamma}(a b)-\sigma_{\gamma}(a) \sigma_{\gamma}(b)\right| \leq 2 \delta+3 \delta=5 \delta .
$$

Let us now show that $\sigma_{\gamma}$ is $\delta^{\prime}$-trace-preserving. Let $s_{i} f s_{j}^{-1} \in(F \cup S)_{ \pm}^{n}$. If $i \neq j$ then

$$
\operatorname{tr}\left(s_{i} f s_{j}^{-1}\right)=\operatorname{tr}\left(\gamma\left(s_{i}\right) \sigma^{\prime}\left(s_{i}^{-1} s_{i} f s_{j}^{-1} s_{j}\right) \gamma\left(s_{j}\right)^{-1}\right)=0,
$$


suppose otherwise, then

$$
\begin{aligned}
\left|\operatorname{tr}\left(\sigma_{\gamma}\left(s_{i} f s_{i}^{-1}\right)\right)-\tau\left(s_{i} f s_{i}^{-1}\right)\right|= & \left|\operatorname{tr}\left(\gamma\left(s_{i}\right) \sigma^{\prime}\left(s_{i}^{-1} s_{i} f s_{i}^{-1} s_{i}\right) \gamma\left(s_{i}\right)^{-1}\right)-\tau\left(s_{i} f s_{i}^{-1}\right)\right| \\
= & \left|\operatorname{tr}\left(\sigma^{\prime}\left(s_{i}^{-1} s_{i} f s_{i}^{-1} s_{i}\right) \gamma\left(s_{i}\right)^{-1} \gamma\left(s_{i}\right)\right)-\tau\left(f s_{i}^{-1} s_{i}\right)\right| \\
\leq & \left.\mid \operatorname{tr}\left(\sigma^{\prime}\left(s_{i}^{-1} s_{i} f s_{i}^{-1} s_{i}\right) \gamma\left(s_{i}\right)^{-1} \gamma\left(s_{i}\right)\right)\right)-\operatorname{tr}\left(\sigma^{\prime}\left(s_{i}^{-1} s_{i} f s_{i}^{-1} s_{i}\right)\right) \mid \\
& \quad+\left|\operatorname{tr}\left(\sigma^{\prime}\left(s_{i}^{-1} s_{i} f s_{i}^{-1} s_{i}\right)\right)-\frac{(N-k) \tau\left(f s_{i}^{-1} s_{i}\right)}{N \mathfrak{h}(p)}\right| \\
\leq & (2 \delta+\delta)+\delta<\delta^{\prime}
\end{aligned}
$$

where we used the computation above.

So we have proved

$$
\left|\mathrm{SA}_{p G p}(F, 4 n+5, \delta, d)\right|_{E} \leq\left|\mathrm{SA}_{G}\left(F \cup S, n, \delta^{\prime}, d^{\prime}\right)\right|_{E \cup S} / \frac{\left(\frac{d^{\prime}}{N} !\right)^{k}}{(\lceil\delta d\rceil+1)^{\lceil\delta d\rceil k}}
$$

So

$$
\begin{aligned}
& \frac{\log \left|\mathrm{SA}_{p G p}(F, 4 n+5, \delta, d)\right|_{E}}{d \log d} \leq \frac{\log \left(\left|\mathrm{SA}_{G}\left(F \cup S, n, \delta^{\prime}, d^{\prime}\right)\right| E \cup S / \frac{\left(\frac{d^{\prime}}{N} !\right)^{k}}{(\lceil\delta d]+1)^{|\delta d| k}}\right)}{d \log d} \\
&=\frac{N}{N-k} \frac{\log \left|\mathrm{SA}_{G}\left(F \cup S, n, \delta^{\prime}, d^{\prime}\right)\right|_{E \cup S}}{d^{\prime} \log d} \\
&-k \frac{\log \left(\frac{d^{\prime}}{N}\right) !}{d \log d}+\lceil\delta d\rceil k \frac{\log (\lceil\delta d+1\rceil)}{d \log d}
\end{aligned}
$$

Now for $\varepsilon>0$ arbitrary and $d$ sufficiently large we have $\frac{1}{\log d} \leq \frac{1}{\log d^{\prime}}+\frac{\varepsilon}{\log d^{\prime}}$ so the left hand side is at most

$$
\frac{N}{N-k}(1+\epsilon) \frac{\left.\log \left|\mathrm{SA}_{G}\left(F \cup S, n, \delta^{\prime}, d^{\prime}\right)\right|_{E \cup S}\right)}{d^{\prime} \log d^{\prime}}-k \frac{\log \left(\frac{d^{\prime}}{N}\right) !}{d \log d}+\lceil\delta d\rceil k \frac{\log (\lceil\delta d+1\rceil)}{d \log d}
$$

By Stirlings approximation

therefore

$$
\log \left(\frac{d^{\prime}}{N}\right) !=\frac{d^{\prime}}{N} \log \frac{d^{\prime}}{N}+\frac{d^{\prime}}{N}+O\left(\log \frac{d^{\prime}}{N}\right)
$$

$$
s_{p G p, E}(F, 2 n+5, \delta) \leq \frac{N}{N-k}(1+\varepsilon) \limsup _{d \rightarrow \infty} s_{G, E \cup S}\left(F \cup S, n, \delta^{\prime}, d^{\prime}\right)+\frac{k}{N}+k \delta
$$

which gives

$$
\begin{aligned}
s_{p G p, E}(F, 4 n+5) & \leq \frac{1}{\mathfrak{h}(p)}(1+\varepsilon) s_{G, E \cup S}(F \cup S, n)+\frac{k}{N} \\
& =\frac{1}{\mathfrak{h}(p)}(1+\varepsilon) s_{G, E \cup S}(F \cup S, n)+1-\frac{1}{\mathfrak{h}(p)}
\end{aligned}
$$

From here it is clear that

$$
s(p G p) \leq \frac{1}{\mathfrak{h}(p)} s(G)+1-\frac{1}{\mathfrak{h}(p)} .
$$

The same proof works with $\liminf \operatorname{in}_{d \rightarrow \infty}$ and $\lim _{d \rightarrow \omega}$ instead of $\lim \sup _{d \rightarrow \infty}$.

Next we prove the other direction in the general case: 
Lemma 32. $s(G) \leq \mathfrak{h}(p) s(p G p)+1-\mathfrak{h}(p)$ and similarly for $\underline{s}$ and $s_{\boldsymbol{\omega}}$.

Proof. Let $S=\left\{s_{1}, s_{2}, \ldots, s_{k}\right\} \subset \llbracket G \rrbracket$ be such that

$$
s_{i}^{-1} s_{i} \leq p \text { and } \sum_{i=1}^{k} s_{i} s_{i}^{-1}=1-p .
$$

and let $E, F \subset \llbracket p G p \rrbracket$ be finite subsets. We may assume that $p \in E \cap F$ and $s_{i} s_{i}^{-1}, s_{i}^{-1} s_{i} \in E \cap F$ for all $i$.

Let $\sigma \in \mathrm{SA}_{G}(F \cup S, n+4, \delta, d)$ and assume that $d$ is large enough so $d^{\prime}:=\lfloor\mathfrak{h}(p) d\rfloor>$ $\delta^{-1}$. Let $B_{0}=\operatorname{Fix} \sigma(p)$ so

$$
\left|\frac{\left|B_{0}\right|}{d}-\mathfrak{h}(p)\right|<\delta
$$

Thus we may arbitrarily extend or shrink $B_{0}$ to a subset $B$ such that either $B \subset B_{0}$ or $B \supset B_{0}$ and $|B|=d^{\prime}$ where

$$
\begin{aligned}
\left|\sigma(p)-p_{B}\right| & \leq\left|\sigma(p)-p_{\text {Fix } \sigma(p)}\right|+\left|p_{\text {Fix } \sigma(p)}-p_{B}\right| \\
& =\frac{1}{d}\left|\left\{x \in\{1 \ldots d\} \mid \sigma(p)^{2} x \neq \sigma(p)\right\}\right|+\left|\frac{1}{d}\right| B_{0}|-\mathfrak{h}(p)|+\left|\frac{1}{d}\right| B|-\mathfrak{h}(p)| \\
& <3 \delta .
\end{aligned}
$$

Let $\delta^{\prime}=\frac{20 \delta}{\mathfrak{h}(p)}$. We will show that

$$
\sigma^{\prime}:=\sigma_{\left.\right|_{B}} \in \mathrm{SA}_{p G p}\left(F, n, \delta^{\prime}, d^{\prime}\right) .
$$

Cleary $\sigma^{\prime}$ is well defined, let us show it is $\delta^{\prime}$-multiplicative that is for $a, b \in \boldsymbol{\Sigma} F_{ \pm}^{n}$ with $a b \in \boldsymbol{\Sigma} F_{ \pm}^{n}$ that

$$
\left|\sigma^{\prime}(a b)-\sigma^{\prime}(a) \sigma^{\prime}(b)\right|<\delta^{\prime}
$$

So

$$
\begin{aligned}
\left|\sigma^{\prime}(a b)-\sigma^{\prime}(a) \sigma^{\prime}(b)\right| & =\frac{1}{d^{\prime}} \sum_{x \in B}\left|\left\{x \in B \mid \sigma^{\prime}(a b) x \neq \sigma^{\prime}(a) \sigma^{\prime}(b) x\right\}\right| \\
& =\frac{d}{d^{\prime}}\left|p_{B} \sigma(a b) p_{B}-p_{B} \sigma(a) p_{B} \sigma(b) p_{B}\right| \\
& \leq \frac{\delta}{\mathfrak{h}(p)}(15 \delta+5 \delta)=\frac{20 \delta^{2}}{\mathfrak{h}(p)}<\delta^{\prime}, \text { for } \delta<1
\end{aligned}
$$

We now show $\sigma^{\prime}$ is $\delta^{\prime}$-trace-preserving. Let $a \in F_{ \pm}^{n}$ then

$$
\begin{aligned}
\left|\frac{\mid \text { Fix } \sigma^{\prime}(a) \mid}{d^{\prime}}-\frac{\tau_{G}(a)}{\mathfrak{h}(p)}\right| & =\frac{1}{\mathfrak{h}(p)}\left|\frac{\mid \text { Fix } \sigma^{\prime}(a) \mid}{d^{\prime} / \mathfrak{h}(p)}-\tau_{G}(a)\right| \\
& \leq \frac{1}{\mathfrak{h}(p)}\left|\frac{\mathfrak{h}(p)}{d^{\prime}}-\frac{1}{d}\right|+\frac{1}{\mathfrak{h}(p)}\left|\frac{\left|\operatorname{Fix} \sigma^{\prime}(a)\right|}{d}-\tau_{G}(a)\right| \\
& \leq \frac{1}{\mathfrak{h}(p)}\left(\left|\frac{\left|\operatorname{Fix} p_{B} \sigma(a) p_{B}\right|}{d}-\frac{|\operatorname{Fix} \sigma(a)|}{d}\right|+\left|\operatorname{tr} \circ \sigma(a)-\tau_{G}(a)\right|+\delta^{2}\right) \\
& \leq \frac{1}{\mathfrak{h}(p)}\left(\left|p_{B} \sigma(a) p_{B}-\sigma(a)\right|+\delta+\delta^{2}\right) \\
& \leq \frac{1}{\mathfrak{h}(p)}\left((6 \delta+2 \delta)+\delta+\delta^{2}\right)=\frac{9 \delta+\delta^{2}}{\mathfrak{h}(p)}<\delta^{\prime}, \text { for } \delta<1
\end{aligned}
$$

So $\sigma^{\prime} \in \operatorname{SA}_{p G p}\left(F, n, \delta^{\prime}, d^{\prime}\right)$ as claimed. 
Next we study the map

$$
\begin{aligned}
\operatorname{SA}_{G}(F \cup S, n+4, \delta, d) & \rightarrow \mathrm{SA}_{p G p}\left(F, n, \delta^{\prime}, d^{\prime}\right) \\
\sigma & \mapsto \sigma^{\prime}
\end{aligned}
$$

Note that

$$
\begin{aligned}
\left|\frac{\left|\operatorname{Fix} \sigma\left(s_{i}\right) \sigma\left(s_{i}\right)^{-1}\right|}{d}-\mathfrak{h}\left(s_{i} s_{i}^{-1}\right)\right| & \leq\left|\frac{\left|\operatorname{Fix} \sigma\left(s_{i}\right) \sigma\left(s_{i}\right)^{-1}\right|}{d}-\frac{\left|\operatorname{Fix} \sigma\left(s_{i} s_{i}^{-1}\right)\right|}{d}\right| \\
& +\left|\operatorname{tr} \circ \sigma\left(s_{i} s_{i}^{-1}\right)-\mathfrak{h}\left(s_{i} s_{i}^{-1}\right)\right| \\
& \leq\left|\sigma\left(s_{i}\right) \sigma\left(s_{i}\right)^{-1}-\sigma\left(s_{i} s_{i}^{-1}\right)\right|+\delta \\
& \leq 5 \delta+\delta<6 \delta
\end{aligned}
$$

and for $i \neq j$

$$
\begin{aligned}
\left|\operatorname{ran}\left(\sigma\left(s_{i}\right)\right) \cap \operatorname{ran}\left(\sigma\left(s_{j}\right)\right)\right| & =\left|\left\{x \in\{1, \ldots, d\} \mid \sigma\left(s_{j}\right) \sigma\left(s_{j}\right)^{-1} \sigma\left(s_{i}\right) \sigma\left(s_{i}\right)^{-1} x \neq 0\right\}\right| \\
& \leq d\left|\sigma\left(s_{j}\right) \sigma\left(s_{j}\right)^{-1} \sigma\left(s_{i}\right) \sigma\left(s_{i}\right)^{-1}\right|<9 \delta d
\end{aligned}
$$

so

$$
\begin{aligned}
\left|\operatorname{ran}\left(\sigma\left(s_{i}\right)\right) \cap B\right| & =\left|\left\{x \in\{1, \ldots, d\} \mid p_{B} \sigma\left(s_{i}\right) \sigma\left(s_{i}\right)^{-1} x \neq 0\right\}\right| \\
& \leq d\left|p_{B} \sigma\left(s_{i}\right) \sigma\left(s_{i}\right)^{-1}\right| \leq(3 \delta+5 \delta) d \leq 8 \delta d
\end{aligned}
$$

So for all $d$ sufficiently large

$$
\operatorname{ran}\left(\sigma\left(s_{i}\right)\right) \backslash\left(\bigcup_{j \neq i} \operatorname{ran}\left(\sigma\left(s_{j}\right)\right) \cup B\right)
$$

contains at least $d_{i}:=\left\lfloor\mathfrak{h}\left(s_{i} s_{i}^{-1}\right) d-(14 \delta+9 \delta k) d\right\rfloor$ elements so we can find

$$
A_{i} \subset \operatorname{ran}\left(\sigma\left(s_{i}\right)\right) \backslash\left(\bigcup_{j \neq i} \operatorname{ran}\left(\sigma\left(s_{j}\right)\right) \cup B\right)
$$

be a subset with size $\left|A_{i}\right|=d_{i}$ and $A_{i} \cap A_{j}=\varnothing$ for $i \neq j$ and $A_{i} \cap B=\varnothing$. Similarly we have

$$
\left|\frac{\mid \text { Fix } \sigma\left(s_{i}\right)^{-1} \sigma\left(s_{i}\right)}{d}-\mathfrak{h}\left(s_{i}^{-1} s_{i}\right)\right|<6 \delta
$$

and

$$
\begin{aligned}
\left|\operatorname{dom} \sigma\left(s_{i}\right) \triangle \operatorname{Fix} \sigma\left(s_{i}^{-1} s_{i}\right) \cap B\right| & \leq\left|\left\{x \in\{1 \ldots, d\} \mid P_{B} P_{\mathrm{Fix} \sigma\left(s_{i}^{-1} s_{i}\right)} x \neq \sigma\left(s_{i}\right)^{-1} \sigma\left(s_{i}\right) x\right\}\right| \\
& \leq d\left|P_{B} P_{\mathrm{Fix} \sigma\left(s_{i}^{-1} s_{i}\right)}-\sigma\left(s_{i}\right)^{-1} \sigma\left(s_{i}\right)\right|<d 6 \delta
\end{aligned}
$$

So we can find subsets $B_{i} \subset \operatorname{dom}\left(\sigma\left(s_{i}\right)\right) \cap \operatorname{Fix} \sigma\left(s_{i}^{-1} s_{i}\right) \cap B$ with $\left|B_{i}\right|=d_{i}$. Let $d_{k+1}=d-d^{\prime}-\sum_{i=1}^{k} d_{i}$. Also recall that $\left|\operatorname{Fix} \sigma\left(s_{i} s_{i}^{-1}\right)\right| \leq d\left(\mathfrak{h}\left(s_{i}^{-1} s_{i}\right)+\delta\right)$.

Let $\left|\mathrm{SA}_{G}(F \cup S, n+4, \delta, d)\right|_{E \cup S}^{\prime}$ be the number of elements of $\mathrm{SA}_{G}(F \cup S, n+$ $4, \delta, d)$ where we distinguish elements by their values on $\left\{p_{B} \sigma(f) p_{B} \mid f \in E\right\}$ and $\left\{p_{A_{i}} \sigma\left(s_{i}\right) p_{B_{i}}\right\}$. We have shown with the above computations that

$$
\begin{aligned}
&\left|\mathrm{SA}_{G}(F \cup S, n+4, \delta, d)\right|_{E, S}^{\prime} \\
& \leq\left(\begin{array}{c}
d \\
d^{\prime}, d_{1}, \ldots, d_{k+1}
\end{array}\right)\left|\mathrm{SA}_{p G p}\left(F, n, \delta^{\prime}, d^{\prime}\right)\right|_{E} \\
& \prod_{i=1}^{k}\left(\begin{array}{c}
d\left(\mathfrak{h}\left(s_{i}^{-1} s_{i}\right)+\delta\right) \\
d_{i}
\end{array}\right) \prod_{i=1}^{k} d_{i} !
\end{aligned}
$$


where the former term accounts for the choice of the $A_{i}$ and $B$ and the latter terms for the choice of the $B_{i}$ and $\sigma\left(s_{i}\right)$ respectively.

Now note that for $f \in E$

$$
\left|p_{B} \sigma(f) p_{B}-\sigma(f)\right| \leq(6 \delta+2 \delta)=8 \delta
$$

and similarly for $i \neq j$

$$
\begin{aligned}
\left|p_{A_{i}} \sigma\left(s_{i}\right) p_{B_{i}}-\sigma\left(s_{i}\right)\right| & \leq\left|p_{A_{i}}-\sigma\left(s_{i}\right)^{-1} \sigma\left(s_{i}\right)\right|+\left|p_{B_{i}}-\sigma\left(s_{i}\right) \sigma\left(s_{i}\right)^{-1}\right|+3 \delta \\
& \leq(14 \delta+9 k \delta+6 \delta)+(14 \delta+9 k \delta+6 \delta)+\delta+3 \delta, \text { for d sufficiently large } \\
& =(44+18 k) \delta
\end{aligned}
$$

so we can find $\kappa>0$ with $\kappa \rightarrow 0$ as $\delta \rightarrow 0$ so that for a given choice of $p_{B} \sigma(f) p_{B}$ there are $d^{\kappa d}$ choices for $\sigma(f)$ and similarly for $\sigma\left(s_{i}\right)$ (see [DKP2, Lemma 2.5]) so

$$
\begin{aligned}
&\left|\mathrm{SA}_{G}(F \cup S, n+4, \delta, d)\right|_{E, S} \\
& \leq\left(\begin{array}{c}
d \\
d^{\prime}, d_{1}, \ldots, d_{k+1}
\end{array}\right)\left|\mathrm{SA}_{p G p}\left(F, n, \delta^{\prime}, d^{\prime}\right)\right|_{E} \\
& \prod_{i=1}^{k}\left(\begin{array}{c}
d\left(\mathfrak{h}\left(s_{i}^{-1} s_{i}\right)+\delta\right) \\
d_{i}
\end{array}\right) \prod_{i=1}^{k}\left(d_{i} !\right) d^{\kappa d|E \cup S|} .
\end{aligned}
$$

Therefore

$$
\begin{aligned}
\log \mid \mathrm{SA}_{G}(F \cup S, & +4, \delta, d)\left.\right|_{E, S} \leq \log \frac{d !}{d^{\prime} ! d_{k+1} !}+\log \left|\mathrm{SA}_{p G p}\left(F, n, \delta^{\prime}, d^{\prime}\right)\right|_{E} \\
& +\sum_{i=1}^{k} \log \frac{d\left(\mathfrak{h}\left(s_{i}^{-1} s_{i}\right)+\delta\right) !}{d_{i} !\left(d\left(\mathfrak{h}\left(s_{i}^{-1} s_{i}\right)+\delta\right)-d_{i}\right) !}+\kappa d|E \cup S| \log d
\end{aligned}
$$

hence

$$
\log \left|\mathrm{SA}_{G}(F \cup S, n+4, \delta, d)\right|_{E, S}+\log d^{\prime} ! \leq \log d !+\log \left|\mathrm{SA}_{p G p}\left(F, n, \delta^{\prime}, d^{\prime}\right)\right|_{E}+\varepsilon(\delta, d) .
$$

with

$$
\varepsilon(\delta, d):=\sum_{i=1}^{k} \log \frac{d\left(\mathfrak{h}\left(s_{i}^{-1} s_{i}\right)+\delta\right) !}{d_{i} !\left(d\left(\mathfrak{h}\left(s_{i}^{-1} s_{i}\right)+\delta\right)-d_{i}\right) !}+\kappa d|E \cup S| \log d
$$

so since $\lim _{d \rightarrow \infty} \frac{\log d^{\prime} !}{\mathrm{d} \log d}=\mathfrak{h}(p)$ and $\inf _{\delta} \lim _{d \rightarrow \infty} \varepsilon(\delta, d)=0$ we obtain finally

$$
s_{G, E \cup S}(F \cup S, n+4)+\mathfrak{h}(p) \leq 1+\inf _{\delta>0} \limsup _{d \rightarrow \infty} \frac{\left|\operatorname{SA}_{p G p}\left(F, n, \delta^{\prime}, d^{\prime}\right)\right|_{E}}{d \log d} .
$$

Using [DKP2, Lemma 2.13]

$$
s_{G, E \cup S}(F \cup S, n+4)+\mathfrak{h}(p) \leq 1+\mathfrak{h}(p) s_{p G p, E}(F, n)
$$

and we deduce

$$
s(G) \leq \mathfrak{h}(p) s(p G p)+1-\mathfrak{h}(p) .
$$

The same proof works with $\liminf _{d \rightarrow \infty}$ and $\lim _{d \rightarrow \omega}$ instead of $\lim \sup _{d \rightarrow \infty}$.

Finally we deduce the scaling formula:

Proof of Proposition 30. Let $p_{n} \leq p \leq q_{n}$ be projections with $\mathfrak{h}\left(p_{n}\right), \mathfrak{h}\left(q_{n}\right) \in \mathbb{Q}$ and $\left|p_{n}-q_{n}\right| \rightarrow 0$.

$$
\mathfrak{h}(p) s(p G p) \leq \mathfrak{h}\left(p_{n}\right) s\left(p_{n} G p_{n}\right)+\mathfrak{h}(p)-\mathfrak{h}\left(p_{n}\right)
$$

by the previous lemma so $\liminf _{n \rightarrow \infty} s\left(p_{n} G p_{n}\right) \geq s(p G p)$. Similarly

$$
\mathfrak{h}\left(q_{n}\right) s\left(q_{n} G q_{n}\right) \leq \mathfrak{h}(p) s(p G p)+\mathfrak{h}\left(q_{n}\right)-\mathfrak{h}(p)
$$


by the previous lemma so $\lim \sup _{n \rightarrow \infty} s\left(q_{n} G q_{n}\right) \leq s(p G p)$. However the two lemmas combined imply

$$
s(G)=\mathfrak{h}\left(p_{n}\right) s\left(p_{n} G p_{n}\right)+1-\mathfrak{h}\left(p_{n}\right) .
$$

so

and

$$
\limsup _{n \rightarrow \infty} s\left(p_{n} G p_{n}\right)=\frac{1}{\mathfrak{h}(p)}(s(g)-1)+1
$$

$$
s(G)=\mathfrak{h}\left(q_{n}\right) s\left(q_{n} G q_{n}\right)+1-\mathfrak{h}\left(q_{n}\right)
$$

so

Therfore

$$
\liminf _{n \rightarrow \infty} s\left(q_{n} G q_{n}\right)=\frac{1}{\mathfrak{h}(p)}(s(G)-1)+1 .
$$

$$
s(p G p)=\frac{1}{\mathfrak{h}(p)}(s(G)-1)+1
$$

and the same argument works for $\underline{s}$ and $s_{\omega}$.

So

$$
\underline{s}(G)=s(G) \Leftrightarrow \underline{s}(p G p)=s(p G p)
$$

so $G$ is $s$-regular if and only if $p G p$ is $s$-regular.

\section{Applications of the Correspondence principle and the proof of}

\section{THEOREM 1}

Since the work of $\overline{D y e}$, orbit equivalence relations have been used to prove results in group theory on several occasions, with the Bernoulli action $\Gamma \curvearrowright X_{0}^{\Gamma}$ serving as a link. A recent example is the use of the Gaboriau-Lyons theorem GL (that the orbit relation of $\Gamma \curvearrowright[0,1]^{\Gamma}$ of a non amenable group $\Gamma$ contains a nonamenable subtreeing) as a way to replace the assumption "contains a nonamenable free group" on $\Gamma$ by " $\Gamma$ is non amenable". The 'correspondence principle' discussed here is a straightforward but useful extension of this well-known idea from groups to groupoids.

For example let us prove the following result using the principle:

Proposition 33. Let $G \curvearrowright X$ be a pmp action of an amenable pmp groupoid $G$. Then $s(G)=s(G \ltimes X)$. In particular $s(G \ltimes X)$ doesn't depend on the action.

Let us first observe that the measure of the set of finite orbits of an action $G \curvearrowright X$ doesn't depend on the action:

Lemma 34. If $G \curvearrowright X$ is an essentially free pmp action of a pmp groupoid $G$ and $D \subset X$ is a fundamental domain for the set of finite orbits of $G$ in $X$, then

$$
\mu(D):=\int_{G^{0}} \frac{1}{\left|G_{e}\right|} \mathrm{d} \mathfrak{h}(e)
$$

so $\mu(D)$ depends on $G$ only.

Proof. Let

$$
X_{\mathrm{f}}:=\{x \in X|| G x \mid<\infty\} .
$$

Since $G \curvearrowright X$ is free $|G x|=\left|G_{\mathbf{r}(x)}\right|$ almost surely so

$$
\mu^{e}\left(X_{\mathrm{f}}^{e}\right)=\left\{\begin{array}{l}
0 \text { if }\left|G_{e}\right|=\infty \\
1 \text { else }
\end{array}\right.
$$


for ae $e \in G^{0}$ and if $D \subset X_{\mathrm{f}}$ is a fundamental domain then if $e_{1}, e_{2} \ldots, e_{n}$ are the units isomorphic to $e$ we have

$$
\sum_{i=1}^{n} \mu^{e_{i}}\left(D^{e_{i}}\right)=\frac{n}{\left|G_{e}\right|}
$$

therefore

$$
\mu(D)=\int_{G^{0}} \frac{1}{\left|G_{e}\right|} \mathrm{d} \mathfrak{h}(e) .
$$

using invariance of $\mu$.

Remark 35. An analog of the so-called "fixed price problem" Gab for $s$ is the question of whether $s(G \ltimes X)$ depends on $G$ only and not on the essentially free pmp action $G \curvearrowright X$. This "fixed sofic dimension problem" holds for example for groupoids with fixed price 1 (=all their free pmp actions have cost 1 ) provided all their free pmp actions are sofic, but it is open in general. Also open is whether a groupoid is sofic if and only if all its free pmp actions are sofic.

Proof of Proposition 33. Since the Bernoulli action $G \curvearrowright[0,1]^{G}$ is essentially free $G \ltimes[0,1]^{G}$ is a pmp equivalence relation so by Theorem 28] and DKP1, Corollary $5.2]$

$$
s(G)=s\left(G \ltimes[0,1]^{G}\right)=1-\mu(D)=1-\int_{G^{0}} \frac{1}{\left|G_{e}\right|} \mathrm{d} \mathfrak{h}(e)
$$

where $D$ is the fundamental domain of the set of finite classes of the amenable pmp equivalence relation $G \ltimes[0,1]^{G}$.

If $G \curvearrowright X$ is a pmp action then the action $G \ltimes X \curvearrowright[0,1]^{G \ltimes X}$ is pmp and essentially free so by Theorem 28

$$
s(G \ltimes X)=s\left((G \ltimes X) \ltimes[0,1]^{G \ltimes X}\right) .
$$

Now the measure isomorphism

$$
\begin{aligned}
(G \ltimes X) \ltimes[0,1]^{G \ltimes X} & \rightarrow G \ltimes\left(X \times[0,1]^{G \ltimes X}\right) \\
((s, x), y) & \mapsto(s,(x, y))
\end{aligned}
$$

is an isomorphism of pmp equivalence relations, where the action $G \curvearrowright X \times[0,1]^{G \ltimes X}$ is diagonal and $G \curvearrowright[0,1]^{G \ltimes X}$ on the first coordinate.

So

$$
\begin{aligned}
s(G \ltimes X) & =s\left(G \ltimes\left(X \times[0,1]^{G \ltimes X}\right)\right) \\
& =1-\mu\left(D^{\prime}\right) \\
& =1-\int_{G^{0}} \frac{1}{\left|G_{e}\right|} \mathrm{d} \mathfrak{h}(e) .
\end{aligned}
$$

where $D^{\prime}$ is the is the fundamental domain of the set of finite classes of the amenable pmp equivalence relation $G \curvearrowright X \times[0,1]^{G \ltimes X}$. So

$$
s(G)=s(G \ltimes X) .
$$

Another example is the proof that the invariance of $s$ under orbit equivalence (Theorem 9) established in [DKP1, Theorem 4.1] is equivalent to the statement for groupoids in [DKP2, Theorem 2.1].

Theorem 36. Let $G$ be a pmp groupoid, E, $F$ be transversally generating sets, then $s(E)=s(F), \underline{s}(E)=\underline{s}(F)$ and $s^{\omega}(E)=s^{\omega}(F)$. 
Proof. If $F$ is a transversally generating set of $G$ (which we assume to have infinite fibers) then $F \cup\left\{p_{B_{0}}, p_{B_{1}}\right\}$ (as defined in Lemma 15) is a transversally generating set of the pmp equivalence relation $G \ltimes\{0,1\}^{G}$. By Theorem 28

$$
s(F)=s\left(F \cup\left\{p_{B_{0}}, p_{B_{1}}\right\}\right)
$$

so by Theorem 9 we have

$$
s(E)=s\left(E \cup\left\{p_{B_{0}}, p_{B_{1}}\right\}\right)=s\left(F \cup\left\{p_{B_{0}}, p_{B_{1}}\right\}\right)=s(F) .
$$

The same applies to $\underline{s}$ and $s_{\omega}$.

We conclude with the proof Theorem [ 1 which is our main illustration of the correspondence principle.

Proof of Theorem 1, Let $\tilde{G}_{i}:=G_{i \mid G_{3}^{0}}$ and $\tilde{G}:=G_{\mid G_{3}^{0}}=\tilde{G}_{1} \star_{G_{3}} \tilde{G}_{2}$ then for every essentially free pmp action $\tilde{G} \curvearrowright X$ of $\tilde{G}$ we have

$$
\begin{aligned}
s(G)-1= & \mathfrak{h}\left(G_{3}^{0}\right)\left(s\left(\tilde{G}_{1} \star_{G_{3}} \tilde{G}_{2}\right)-1\right) \text { by Proposition 30 } \\
\geq & \mathfrak{h}\left(G_{3}^{0}\right)\left(s\left(\tilde{G}_{1} \star_{G_{3}} \tilde{G}_{2} \ltimes X\right)-1\right) \text { by Proposition 25] } \\
= & \mathfrak{h}\left(G_{3}^{0}\right)\left(s\left(\left(\tilde{G}_{1} \ltimes X_{\mid 1}\right) \star_{\left(G_{3} \ltimes X_{\mid 3}\right)}\left(\tilde{G}_{2} \ltimes X_{\mid 2}\right)\right)-1\right) \text { by Lemma } 18 \\
= & \mathfrak{h}\left(G_{3}^{0}\right)\left(s\left(R_{1} \star_{R_{3}} R_{1}\right)-1\right) \text { where } R_{i}:=\tilde{G}_{i} \ltimes X_{\mid i} \text { are pmp } \\
& \quad \text { equivalence relations by Lemma 14] } \\
= & \mathfrak{h}\left(G_{3}^{0}\right)\left(s\left(R_{1}\right)+s\left(R_{2}\right)-s\left(R_{3}\right)-1\right) \text { by [DKP1, Theorem 1.2] } \\
= & \mathfrak{h}\left(G_{3}^{0}\right)\left(s\left(R_{1}\right)+s\left(R_{2}\right)-s\left(G_{3}\right)-1\right) \text { by Proposition 33 }
\end{aligned}
$$

If $\tilde{G} \curvearrowright X$ is Bernoulli then

$$
s\left(\tilde{G}_{1} \star_{G_{3}} \tilde{G}_{2}\right)=s\left(\tilde{G}_{1} \star_{G_{3}} \tilde{G}_{2} \ltimes X\right)
$$

by Theorem 28 and $\tilde{G}_{i} \curvearrowright X_{\mid i}$ for $i=1,2$ are (isomorphic to) Bernoulli actions by Lemma 13, Therefore $s\left(R_{i}\right)=s\left(\tilde{G}_{i}\right), i=1,2$ by Theorem 28. Then

$$
\begin{aligned}
s(G)-1 & =\mathfrak{h}\left(G_{3}^{0}\right)\left(s\left(R_{1}\right)+s\left(R_{2}\right)-s\left(G_{3}\right)-1\right) \\
& =\mathfrak{h}\left(G_{3}^{0}\right)\left(s\left(\tilde{G}_{1}\right)+s\left(\tilde{G}_{2}\right)-s\left(G_{3}\right)-1\right) \text { by Lemma } 13 \text { and } \\
& =\mathfrak{h}\left(G_{3}^{0}\right)\left(s\left(\tilde{G}_{1}\right)-1\right)+\mathfrak{h}\left(G_{3}^{0}\right)\left(s\left(\tilde{G}_{2}\right)-1\right)-\mathfrak{h}\left(G_{3}^{0}\right)\left(s\left(G_{3}\right)-1\right) \\
& =\mathfrak{h}\left(G_{1}^{0}\right)\left(s\left(G_{1}\right)-1\right)+\mathfrak{h}\left(G_{2}^{0}\right)\left(s\left(G_{2}\right)-1\right)-\mathfrak{h}\left(G_{3}^{0}\right)\left(s\left(G_{3}\right)-1\right) \\
& \quad \text { by Proposition } 30 \\
& =\mathfrak{h}\left(G_{1}^{0}\right) s\left(G_{1}\right)+\mathfrak{h}\left(G_{2}^{0}\right) s\left(G_{2}\right)-\mathfrak{h}\left(G_{3}^{0}\right) s\left(G_{3}\right)-\mathfrak{h}\left(G_{1}^{0}\right)-\mathfrak{h}\left(G_{2}^{0}\right)+\mathfrak{h}\left(G_{3}^{0}\right) \\
& =\mathfrak{h}\left(G_{1}^{0}\right) s\left(G_{1}\right)+\mathfrak{h}\left(G_{2}^{0}\right) s\left(G_{2}\right)-\mathfrak{h}\left(G_{3}^{0}\right) s\left(G_{3}\right)-1
\end{aligned}
$$

(We note that the inequality $s\left(G_{1} *_{G_{3}} G_{2}\right) \leq s\left(G_{1}\right)+s\left(G_{2}\right)-s\left(G_{3}\right)$ could also be proved by a direct argument.)

\section{REFERENCES}

[BCG] Biane, P., Capitaine, M., Guionnet, A. Large deviation bounds for matrix Brownian motion. Invent. Math., 152(2), 433-459. (2003)

[Bow] L. Bowen. Measure conjugacy invariants for actions of countable sofic groups. J. Amer. Math. Soc. 23 (2010), 217-245.

[Bow2] — Entropy theory for sofic groupoids I: the foundations arXiv:1210.1992 
[BDJ] N.P. Brown, K. Dykema, and K. Jung, Free entropy dimension in amalgamated free products, Proc. London Math. Soc. 97 (2008), no. 2, 339-367.

[Dye] H.A. Dye, On groups of measure preserving transformations. I, Amer. J. Math. 81 (1959), no. 1, 119-159.

[DKP1] K. Dykema, D. Kerr, M. Pichot, Orbit equivalence and sofic approximation, arXiv:1102.2556

[DKP2] _ Sofic dimension for discrete measure groupoids, arXiv:1111.2842

[EL] G. Elek and G. Lippner, Sofic equivalence relations, J. Funct. Anal. 258 (2010), no. 5, 1692-1708.

[FSZ] Feldman, J., Sutherland, C. E., Zimmer, R. J. Subrelations of ergodic equivalence relations. Erg. Th. Dyn. Sys., 1988, 9(02), 239-269.

[Gab] D. Gaboriau, Coût des relations d'équivalence et des groupes, Invent. Math. 139 (2000), no. 1, 41-98.

[GL] Gaboriau, D., Lyons, R. A measurable-group-theoretic solution to von Neumann's problem. Invent. Math., 177(3), (2009) 533-540.

[Jun1] Jung, K. . A free entropy dimension lemma. Pacific J. Math. (2003), 177, 265-271.

[Jun2] — The free entropy dimension of hyperfinite von Neumann algebras. Trans. AMS, 34. (2003).

[MS] Mineyev, I., Shlyakhtenko, D. Non-microstates free entropy dimension for groups, GAFA (December 2005), 1-13.

[OW] D. S. Ornstein and B. Weiss. Entropy and isomorphism theorems for actions of amenable groups. J. Analyse Math. 48 (1987), 1-141.

[Oz] N. Ozawa, Hyperlinearity, sofic groups and applications to group theory, Notes from a 2009 talk available at http://people.math.jussieu.fr/ pisier/taka.talk.pdf

[Pe] V. Pestov, Hyperlinear and sofic groups: a brief guide. Bull. Symbolic Logic 14 (2008), no. 4, 449-480.

[Ram] Ramsay, A. Virtual groups and group actions. Advances in Mathematics, 322 (1971), 253322.

[Shl] D. Shlyakhtenko, Microstates free entropy and cost of equivalence relations, Duke Math. J. 118 (2003), no. 3, 375-426.

[Voi91] D. Voiculescu, Limit laws for random matrices and free products, Invent. Math. 104 (1991), no. 1, 201-220.

[Voi96] - The analogues of entropy and of Fisher's information measure in free probability theory III: The absence of Cartan subalgebras, Geom. Funct. Anal. 6 (1996), no. 1, 172-199.

[Voi98] _ A strengthened asymptotic freeness result for random matrices with applications to free entropy, Int. Math. Res. Not. 1998 (1998), no. 1, 41-63. 